Supporting Information

\title{
Techno-economic Feasibility and Spatial Analysis of Thermochemical Conversion Pathways for Regional Poultry Waste Valorization
}

Raaj R. Bora ${ }^{1}$, Yanqiu Tao ${ }^{1}$, Johannes Lehmann², ${ }^{3}$, Jefferson W. Tester ${ }^{1,3}$, Ruth E. Richardson ${ }^{3}$,

${ }^{4}$, Fengqi You ${ }^{1,3, *}$

${ }^{1}$ Robert Frederick Smith School of Chemical and Biomolecular Engineering, Cornell University, Ithaca, New York 14853, USA

${ }^{2}$ Soil and Crop Sciences, School of Integrated Plant Sciences, College of Agriculture and Life Sciences, Cornell University, Ithaca, New York 14853, USA

${ }^{3}$ Atkinson Center for a Sustainable Future, Cornell University, Ithaca, New York 14853, USA

${ }^{4}$ School of Civil and Environmental Engineering, Cornell University, Ithaca, New York 14853, USA

This file contains: 42 pages, 5 sections, 22 tables and 5 figures.

* Corresponding author. E-mail: fengqi.you@cornell.edu 


\section{$\underline{\text { List of Sections }}$}

Section 1. Simulation cases

- Section 1.1. Slow pyrolysis

- Section 1.2. Fast pyrolysis

- Section 1.3. Hydrothermal liquefaction

- Section 1.4. Gasification

- Section 1.5. Other parameters

Section 2. Spatial analysis

- Section 2.1. Bio-oil upgrading

- Section 2.2. Biochar distribution and application

- Section 2.3. Economic considerations

Section 3. Economic results

- Section 3.1. Equipment costs and NPV calculation Section 3.2. Sensitivity analysis results

Section 4. Spatial analysis results

- Section 4.1. Spatial distribution of soil phosphorus contents does not constrain application

Section 5. Additional information and data

\section{$\underline{\text { List of Tables }}$}

- Table S1. Input poultry litter elemental composition.

- Table S2. Names, operating parameters and modeling units used for the important components in the Aspen Plus simulations

- Table S3. Description of scenarios for spatial analysis in terms of number of plants, technology and capacity

- Table S4. Product phase distribution for the four thermochemical technologies (slow pyrolysis, fast pyrolysis, gasification and hydrothermal liquefaction).

- Table S5. Equipment price distribution for fast pyrolysis technology.

- Table S6. Equipment price distribution for slow pyrolysis technology.

- Table S7. Equipment price distribution for gasification technology.

- Table S8. Equipment price distribution for hydrothermal liquefaction technology.

- Table S9. Price of the important utilities. 
- Table S10. Product market prices used for the revenue calculations.

- Table S11. Revenue generation distribution for the nine cases considered.

- Table S12. NPV calculation for FP-SELL case.

- Table S13. NPV calculation for SP-CHAR case.

- Table S14. NPV calculation for SP-COMB case.

- Table S15. GWP 100-year factors of some of the chemicals involved in the simulations.

- Table S16. Net present value (NPV) and greenhouse gas (GHG) inventory results for the nine considered cases.

- Table S17. Syngas production potential through slow pyrolysis for the 14 CAFOs and the corresponding power that can be generated.

- Table S18. Syngas production potential through fast pyrolysis for the 14 CAFOs and the corresponding power that can be generated.

- Table S19. Details and poultry numbers in each CAFO for NYS.

- Table S20. Distribution of poultry numbers in NYS based on counties.

- Table S21. Parameters and their values, units and meaning for the spatial analysis.

- Table S22. List of considered existing refinery equipment that is capable of processing certain amounts of produced bio-oil.

\section{$\underline{\text { List of Figures }}$}

- Figure S1. Schematic process flow diagrams

- Figure S2. Map containing distribution, names, and annual poultry litter generation amounts (ton/year) for the CAFOs in NYS, as well as existing crude refineries near the State

- Figure S3. Trade-off between economic and environmental performance illustrated through the plot of NPV against greenhouse gas emissions for the nine cases

- Figure S4. Sensitivity analysis results

- Figure S5. Spatial distribution of phosphorus in New York State (NYS).

- Figure S6. Annualized economic breakdown 


\section{SIMULATION CASES AND PARAMETERS}

The major thermochemical technologies analyzed in the study are pyrolysis, gasification and hydrothermal liquefaction (HTL). Pyrolysis can be tuned based on reaction temperature and residence time to produce two useful products in the absence of oxygen. ${ }^{1}$ With fast pyrolysis (FP) ( $500^{\circ} \mathrm{C}$ or lesser), the major product is bio-oil which can be further upgraded to green gasoline and diesel, whereas with slow pyrolysis (SP) $\left(400-600^{\circ} \mathrm{C}\right)$, it is biochar, a soil amendment with potential for long-term carbon sequestration..$^{2-3}$ Gasification is a more mature thermochemical technology, with many large-scale coal processing plants operational owing to the benefits of the syngas product as compared to conventional combustion. ${ }^{4} \mathrm{HTL}\left(300-450^{\circ} \mathrm{C}\right)$, unlike the others, is a novel technology which requires no drying of the feedstock, as it use subcritical water as the reaction medium. ${ }^{5-7}$

The important parameters in the simulations include operating conditions (pressure, temperature, flow rate) for each equipment unit, kinetic and thermodynamic parameters (reaction rates, product distribution, phase equilibrium and separation, heat recovery), as well as various ratios and efficiency considerations (equivalence ratio, conversion efficiency, product ratio). However, the values and assumptions associated with each of these parameters are different for each technology, and this is further explained through Table S1, Figure S1 and the following subsections. The input feed composition was derived after going through multiple papers on thermochemical treatment of poultry litter. ${ }^{8-10}$ Importantly, the moisture content of the feed (22.81\%) and the other elemental concentration reflects the average poultry litter composition in the northeastern states of USA, which includes NYS. ${ }^{11}$ The operating conditions for the simulations for each of the technologies were based on multiple papers and government reports and have been summarized in Table S2. ${ }^{12-15}$ The operating conditions were optimized to aid in processing poultry litter, though it has not been explicitly mentioned in the manuscript. First, the

selection of initial operating parameters was done after going through multiple experimental studies. Additionally, during the simulations, an effort was made to continuously update certain operating parameters to ensure the best performance of the systems. 
Table S1. Input poultry litter elemental composition..$^{8-9}$

\begin{tabular}{ll}
\hline Element & Amount (\% w/w) \\
Moisture & 22.81 \\
Ash & 15.33 \\
C & 34.05 \\
H & 4.42 \\
N & 2.89 \\
O & 42.68 \\
S & 0.63 \\
\hline
\end{tabular}

1.1. Slow pyrolysis: The main components of each slow pyrolysis system considered are the dryer, the pyrolysis reactor, the phase separators and other auxiliary equipment such as pumps and compressors. Poultry litter is dried from $22.8 \%$ to $10 \%$ moisture content using a rotary dryer. The output of the dryer is fed to the pyrolysis reactor which in turn produces biochar, bio-oil, and off gas. The bio-oil is considered to be sold to existing nearby refineries owing to its small production quantities. ${ }^{16-17}$ The off-gas has the option to either be combusted or sent to a combined heat and power plant (CHP) in the spatial analysis depending on the scale of the plant. In the SP-COMB case, the biochar is considered to be combusted to produce additional heat and electricity, and to avoid additional transportation. As against this, the biochar is distributed and sold for land application in the SP-CHAR case, and is utilized for the spatial analysis scenarios, too. Unlike the other technologies, the optimum capacity for slow pyrolysis reactors so far has been found to be relatively small (1-2 tons/hour), so multiple parallel reactors are used to model plants with higher flowrates. The feed stream is assumed to go through drying and grinding before splitting into multiple parallel streams. The products from each reactor on separation then find their way into combined product streams.

1.2. Fast pyrolysis: The fast pyrolysis process is modeled in a similar way as the slow pyrolysis process, with only major differences in the reactor scaling and the downstream processing options. ${ }^{18-19}$ The bio-oil has the option of being sold to an existing refinery (FP-SELL) or to be upgraded within a dedicated facility built specifically for hydrotreating and further processing the bio-oil (FP-UPGRADE). The off-gas is treated in a similar manner as that of slow pyrolysis, and 
the biochar is assumed to have the same market value as the biochar from slow pyrolysis too. ${ }^{20}$ The upgrading plant for the bio-oil includes a hydrotreater, multiple separation and distillation units, as well as a hydrogen production unit (Figure S1). ${ }^{9-10,12,21-22}$

1.3. Hydrothermal liquefaction: For HTL, the poultry litter feed is sent to the reactor without the need for drying, and there is the production of four phases - bio-oil phase, a solid hydrochar phase, a gas phase, and an aqueous phase. The aqueous phase consisting of water and organic compounds is sent to an anaerobic digester (AD) to recover the organic contents and ameliorate environmental concerns, as well as to produce valuable biogas. The bio-oil, similar to the case of fast pyrolysis, is either sold to an existing refinery (HP-SELL) or sent to a customized upgrading unit (HP-UPGRADE) with slightly different configurations owing to the differences in the composition and properties of the two bio-oils. The gaseous phase is not considered useful owing to the high $\mathrm{CO}_{2}$ levels, but the hydrochar is considered to be sold as a soil amendment without the carbon storage abilities present in biochar. ${ }^{13-14}$

1.4. Gasification: The gasification model consists of the gasification reactor, syngas cleaning unit, energy production unit and other auxiliary equipment. ${ }^{15}$ The major products of the process are the syngas and relatively small amounts of biochar. The three cases for gasification are all based on how the gas phase is processed (Table 1 in the main body). The raw syngas is sent to a gas cleaning unit before undergoing the Fischer-Tropsch synthesis in the GA-FT case. The oil obtained is further sent to a hydroprocessing and separation unit for upgrading into valuable products like gasoline and diesel. ${ }^{23-24}$ The other two cases for gasification involve either combusting the syngas or sending it to a CHP for recovery of both heat and electricity that could be used both within the process as well as used to generate revenue. ${ }^{25-27}$ 


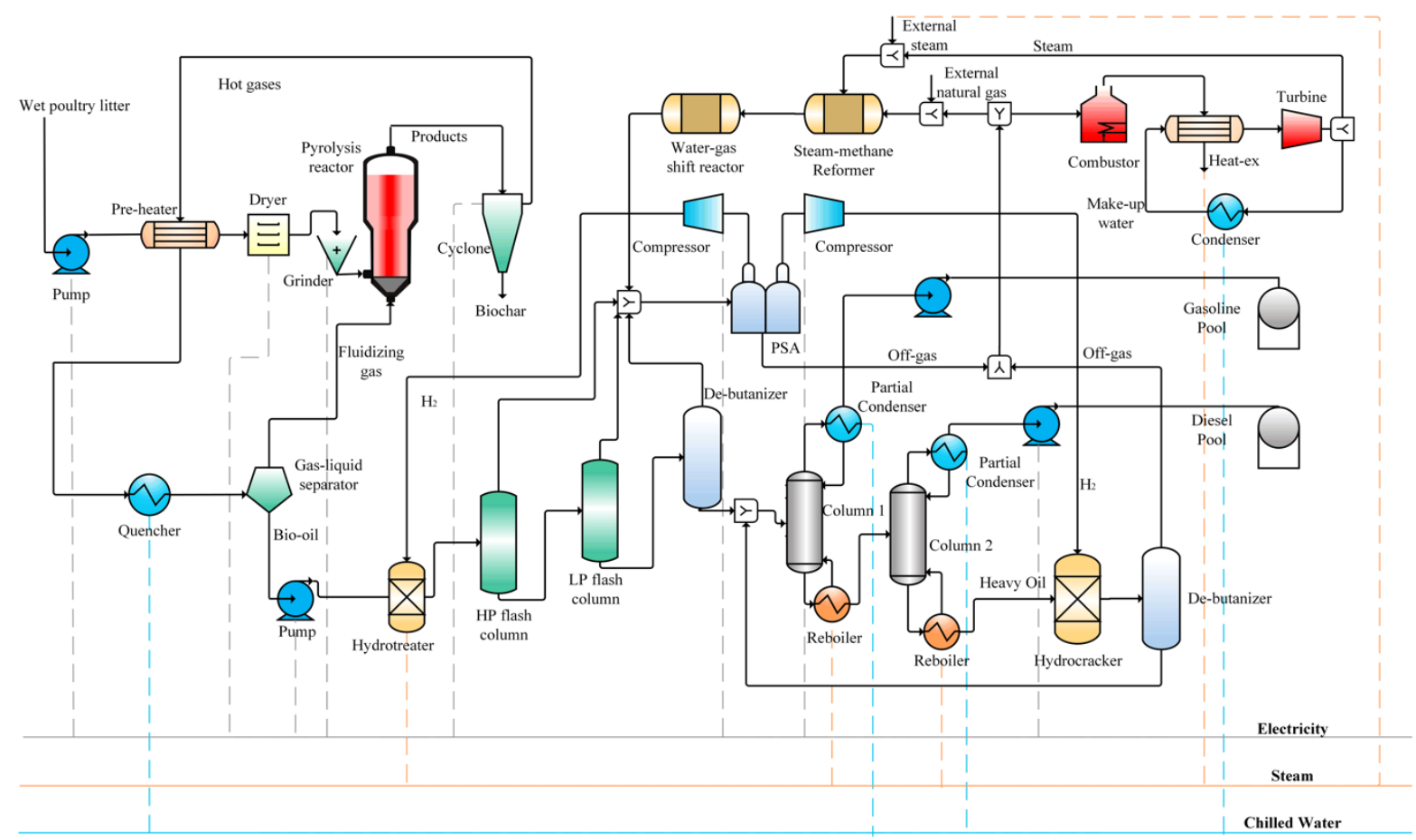

(a)

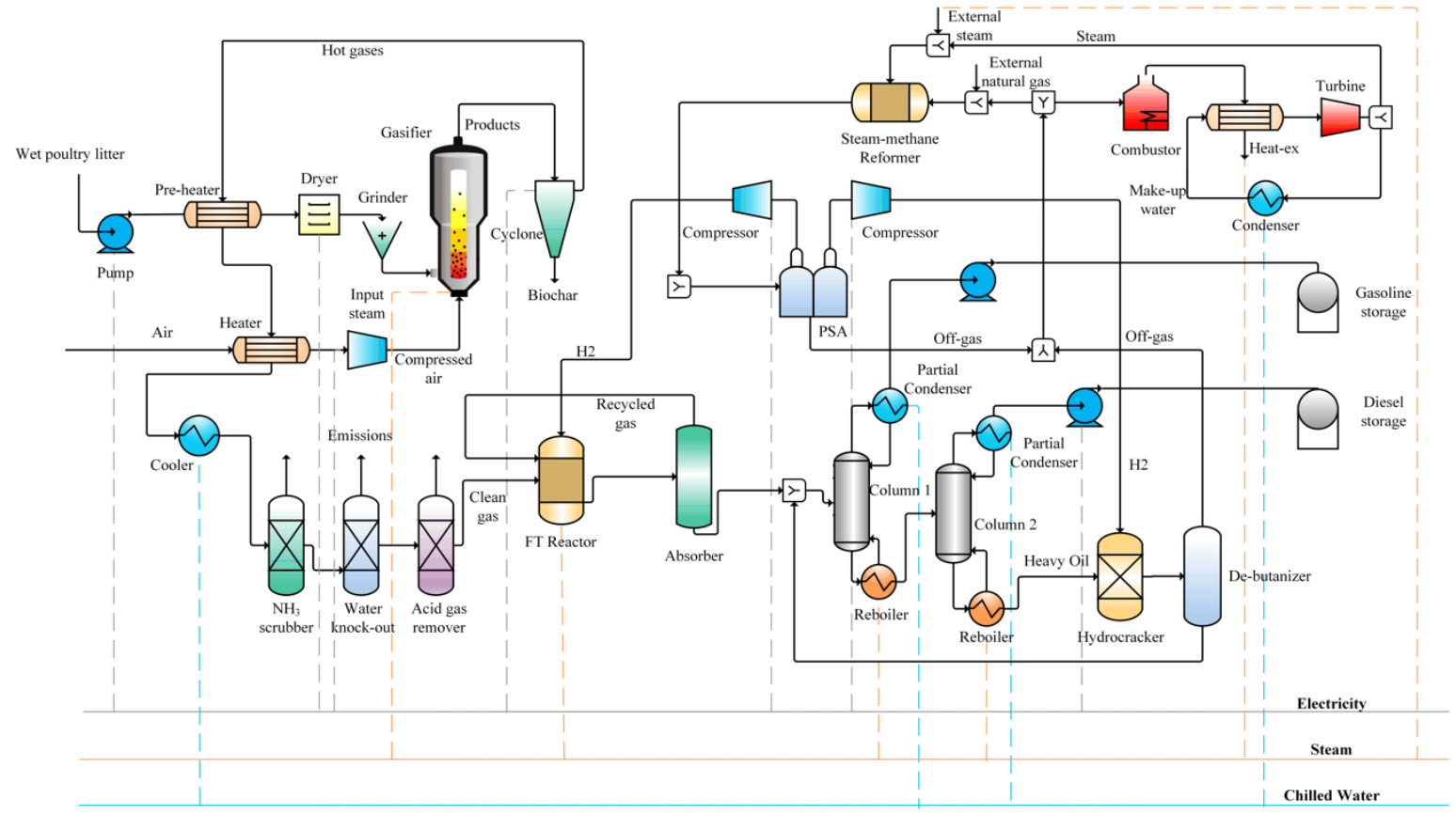

(b) 


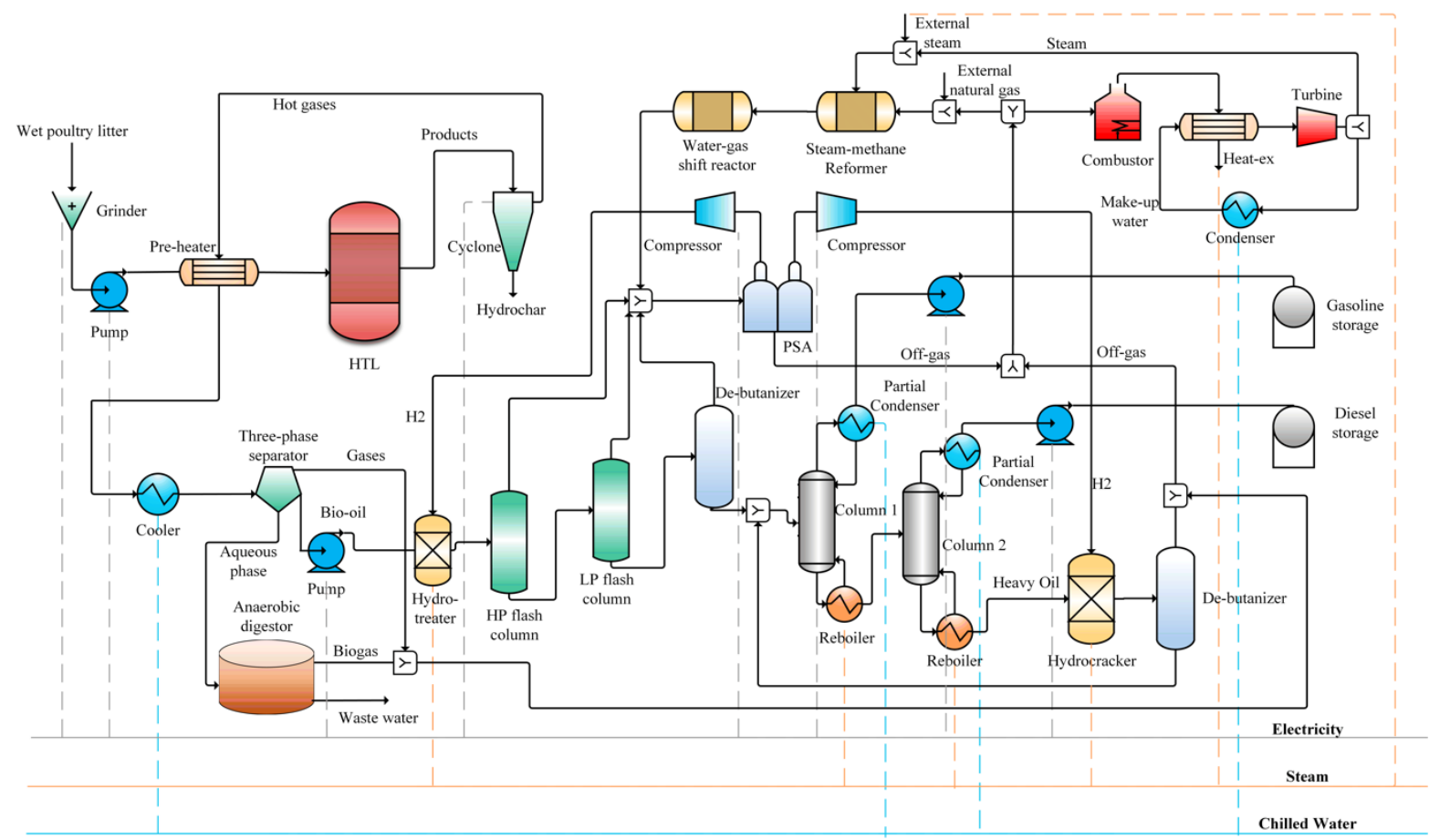

(c)

Figure S1. Schematic process flow diagram of (a) fast pyrolysis pathway with downstream processing of the bio-oil to obtain transportation fuels (FP-UPGRADE) (b) gasification pathway involving Fischer Tropsch process to convert syngas into liquid fuels (GA-FT) (c) hydrothermal liquefaction pathways with upgrading of bio-oil to obtain transportation fuels (HTL-UPGRADE). All the other pathways involve certain parts of these flowsheets. The main utilities are represented through different colors with the gray streams representing electricity, orange streams representing heat, and blue streams representing chilled water.

1.5. Other parameters: In addition to the simulation and economic parameters, there are some other assumptions and parameters which have to be considered and assigned values based on either calculations or literature such as transportation, greenhouse gas emission and sensitivity analysis parameters. The average transportation distances of $50 \mathrm{~km}$ for poultry litter, biochar and bio-oil are used in the economic analysis. However, this value can easily vary based on the distribution of farms and plants and hence the spatial analysis is used to accurately obtain these values in the case study for NYS presented in the Spatial Analysis Section of the main manuscript. The greenhouse gas emission inventory is compiled entirely based on the simulations and certain 
literature values. Emissions from transportation, plant operation, direct utility consumption and biochar soil application are incorporated in the results. Furthermore, in order to identify the influential parameters which could have large impacts on the analysis, sensitivity analyses are conducted for both slow pyrolysis and fast pyrolysis cases. The parameters are chosen after identifying the parameters which lead to the highest change in the NPV values, and their range and base case values are derived from the simulations and literature (Table S20).

Table S2. Names, operating parameters and modeling units used for the important components in the Aspen Plus simulations ${ }^{12-15,20,26,28-31}$

\begin{tabular}{|c|c|c|c|c|c|c|}
\hline $\begin{array}{l}\text { Sr. } \\
\text { No. }\end{array}$ & Equipment & $\begin{array}{c}\text { Temperature } \\
\text { (K) }\end{array}$ & $\begin{array}{c}\text { Pressure } \\
\text { (atm) }\end{array}$ & In scenario(s) & Modeled as & Reference \\
\hline 1 & $\begin{array}{l}\text { Gasification } \\
\text { reactor }\end{array}$ & 1573 & 26 & $\begin{array}{l}\text { GA-FT, GA-CHP, } \\
\text { GA-COMB }\end{array}$ & $\begin{array}{l}\text { RGibbs } \\
\text { reactor }\end{array}$ & 15 \\
\hline 2 & $\begin{array}{l}\text { Fast pyrolysis } \\
\text { reactor }\end{array}$ & 773 & 1 & $\begin{array}{l}\text { FP-SELL, FP- } \\
\text { UPGRADE }\end{array}$ & $\begin{array}{l}\text { RStoic } \\
\text { reactor }\end{array}$ & 12,20 \\
\hline 3 & $\begin{array}{l}\text { Slow } \\
\text { pyrolysis } \\
\text { reactor }\end{array}$ & 923 & 1 & SP-COMB & $\begin{array}{l}\text { RStoic } \\
\text { reactor }\end{array}$ & 26,31 \\
\hline 4 & $\begin{array}{l}\text { Hydrothermal } \\
\text { liquefaction } \\
\text { reactor }\end{array}$ & 623 & 200 & SP-CHAR & $\begin{array}{l}\text { RYield } \\
\text { reactor }\end{array}$ & 13,14 \\
\hline 5 & Preheater & 372 & 1 & ALL & HeatX & - \\
\hline 6 & $\begin{array}{l}\text { Air } \\
\text { compressor }\end{array}$ & - & 26 & $\begin{array}{l}\text { GA-FT, GA-CHP, } \\
\text { GA-COMB }\end{array}$ & Compressor & - \\
\hline
\end{tabular}


7 Dryer

8 Hydrotreater

672

700

10

Gasoline

cooler

11 Diesel cooler

283

283

32

87 GA-FT, FP-

GA-FT, GA-CHP, RStoic + GA-COMB, FP- Flash

SELL, FP-

UPGRADE, SP-

COMB, SP-CHAR

172

32 GA-FT, FP-

12 Fischer Tropsch Reactor

13 Steam methane reformer

14 Water-gas shift reactor

473

25 GA-FT

29 GA-FT, FP-

15 Distillation Column

16 Condenser
45 GA-FT, FPUPGRADE, HTLUPGRADE

573

UPGRADE

1123

23 GA-FT, FP-

UPGRADE, HTLUPGRADE

344

GA-FT, FP-
UPGRADE, HTLUPGRADE

UPGRADE, HTLUPGRADE

GA-FT, FP-

UPGRADE, HTLUPGRADE

UPGRADE, HTLUPGRADE

RYield

reactor UPGRADE, HTL- reactor

RYield

13, 20

reactor

RYield

20

reactor

HeatX

HeatX

RStoic

28

reactor

RStoic

13

UPGRADE, HTL-

UPGRADE 
17 Reboiler

18 Combustor
590

823
1.7 GA-FT, FP-

UPGRADE, HTL-

UPGRADE

GA-COMB, SP- RGibbs

COMB

\section{SPATIAL ANALYSIS}

2.1. Bio-oil upgrading: The bio-oil can either be upgraded on-site or sent to an existing crude refinery, and the crude refinery data are derived from the simulations as well as through the U.S. Energy Information Administration (EIA) website. ${ }^{32}$ Since bio-oil from pyrolysis is found to contain approximately $35 \%$ oxygen and $20 \%$ water in our simulations, the quality of the petroleum products could be negatively affected if a crude refinery is to accept too much bio-oil from the pyrolysis biorefinery. ${ }^{33}$ To be conservative, it is assumed that each crude refinery can only accept bio-oil up to $1 \%$ of its capacity from the biorefineries. ${ }^{34}$ As mentioned before, the bio-oil produced from slow pyrolysis is not upgraded at a dedicated facility since the amount and the quality of biooil from slow pyrolysis is not comparable with the bio-oil produced from fast pyrolysis. For onsite upgrading, the minimum capacity of a bio-oil upgrading facility on a fast pyrolysis biorefinery is assumed to be $10 \mathrm{kton} / \mathrm{year}$ based on the profitability analysis conducted in certain studies and by considering the capacities of currently operational refineries. ${ }^{35}$

2.2. Biochar distribution and application: The biochar is to be applied on corn cropland with an application rate derived from the county-wise recommended $\mathrm{P}$ application data for corn croplands as described in Section 4.1. Corn is selected owing to its prominent distribution across NYS unlike other crops which allows for minimization of the transportation distance for biochar. ${ }^{36}$ The corn cropland data are collected from the NASS Cropland Data Layer ${ }^{36}$ and later aggregated from a pixel resolution of 30 meters to a pixel resolution of 7.5 kilometers. In each scenario, the biochar breakeven price is computed for each pixel and presented on the map of NYS. It is worth mentioning that white pixels on the graphs represent absence of corn cropland and hence inability 
to transport and apply biochar on those pixels. The distribution of corn cropland in NYS is consistent with the protected areas and the areas of waterbodies acquired from the New York Protected Areas Database. ${ }^{37}$ Figure S2 and Table S3 provide details about the distribution of the CAFOs in NYS and the scenarios considered in the spatial analysis.

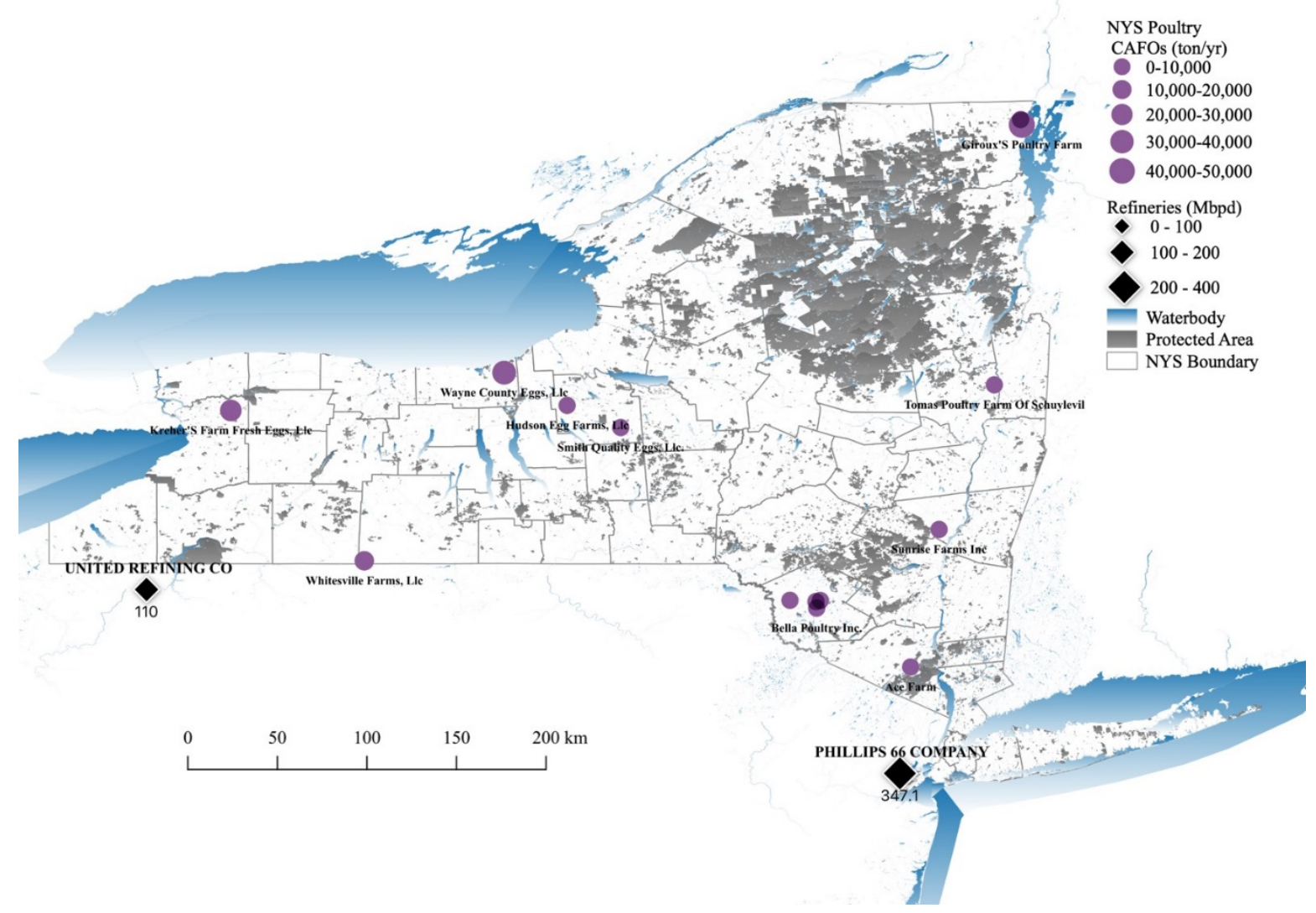

Figure S2. Map containing distribution, names, and annual poultry litter generation amounts (ton/year) for the CAFOs in NYS, as well as existing crude refineries near the State.

Table S3. Description of scenarios for spatial analysis in terms of number of plants, technology and capacity (SP and FP stand for slow pyrolysis and fast pyrolysis, respectively).

\begin{tabular}{cccc}
\hline Scenario name & $\begin{array}{c}\text { No. of } \\
\text { plants }\end{array}$ & Technology & Capacity (kton/year) \\
\hline SP Scenario 1 & 1 & slow & 175.3 \\
SP Scenario 2 - case 1 & 2 & pyrolysis & $150,25.3$ \\
SP Scenario 2 - case 2 & 2 & & $120,55.3$ \\
\hline
\end{tabular}




\begin{tabular}{|c|c|c|c|}
\hline SP Scenario 2 - case 3 & 2 & & $90,85.3$ \\
\hline SP Scenario 3 & 10 & & $\begin{array}{c}\text { 49.5, 39.4, 26.4, 17.3, 9.9, 8.3, 7.6, } \\
7.1,5.4,4.6\end{array}$ \\
\hline FP Scenario 1 & 1 & & 175.3 \\
\hline FP Scenario 2 - case 1 & 2 & & $150,25.3$ \\
\hline FP Scenario 2 - case 2 & 2 & & $120,55.3$ \\
\hline FP Scenario 2 - case 3 & 2 & fast & $90,85.3$ \\
\hline FP Scenario 3 & 8 & pyrolysis & $\begin{array}{c}\text { 49.5, 39.4, 26.4, } 17.3,13.2,9.97,9.9 \\
9.7\end{array}$ \\
\hline
\end{tabular}

2.3. Economic considerations: The annual net revenue is calculated based on the difference between the sum of bio-oil income, biochar income, electricity income and carbon tax income and the sum of capital cost, O\&M cost, poultry litter transportation cost and bio-oil transportation cost along with a fixed biochar price of $\$ 100 /$ ton and without consideration of the biochar transportation cost. All the parameters needed for the calculations are assigned values based on the initial techno-economic calculations. Additionally, in order to show the relationship between the choice of biorefinery locations and the biochar transportation, the biochar breakeven price for all scenarios is also calculated. It is worth mentioning that when annual net revenue turns out to be positive, a negative biochar breakeven price is obtained, and vice versa. Capital cost, O\&M cost, biochar income, bio-oil income, electricity income and carbon tax income are found to be dependent on the capacity and technology choices for the biorefinery, while the poultry litter, biooil and biochar transportation costs are related closely with the location of the biorefineries and corn croplands. The mode of transportation for all products is assumed to be through trucks, and Equation 1 taken from literature ${ }^{38}$ is used to compute the transportation cost (\$/ton).

$$
\text { Transportation cost }=4.1+0.08 \cdot \text { Distance }
$$

Where the distance is calculated in kilometers and ' 0.08 ' is the cost in $\$ /$ ton associated with the transportation of the material whereas ' 4.1 ' is the cost in \$/ton associated with loading and unloading the trucks.

To determine the optimal locations of the biorefineries among the 14 CAFOs, the pairwise route distances between each crude refinery and CAFO, from one CAFO to another, and between 
corn cropland pixels and each CAFO are computed and integrated in the calculation of the breakeven price for biochar.

\section{ECONOMIC RESULTS}

3.1. Equipment costs and NPV calculation: For the four cases with the highest fixed and variable annualized costs (GA-FT, HTL-UPGRADE, FP-UPGRADE and SP-COMB) the equipment costs are also analyzed to help identify the major contributors (Figure 3). Since the magnitude of the equipment costs is large and even a small proportion could make a difference, an attempt is made to include the minor contributors as shown in Figure 3 in the main manuscript and Tables S5-S8. Equipment with similar functions are combined into major groups in each of the pie-charts and the proportion within the group is displayed through three donut charts for each technology (Table S5-S8 contain all the absolute values). Among the major groups, the 'hydroprocessing' fraction consists of hydrotreaters, hydrocrackers and hydrogen production units. The 'separators' include flash vessels, distillation columns, cyclone separators, pressure swing adsorbers (PSA) and other phase separation equipment. The 'others' group within the piecharts consists of an assortment of equipment that could not be associated with any other categories. It consists of equipment such as pumps, compressors, storage tanks, turbines and generators. The NPV results along with their trade-offs with the greenhouse gas emissions are also portrayed in this section (additional discussion in main body of the manuscript). Tables S4-S18 and Figure S3 summarize some of the simulations and economic results.

Table S4. Product phase distribution for the four thermochemical technologies (slow pyrolysis, fast pyrolysis, gasification and hydrothermal liquefaction). ${ }^{10}$

\begin{tabular}{lcccc}
\hline & SP & FP & GA & HTL \\
Gas & 31.57 & 17.14 & 93.60 & 22.95 \\
Oil & 28.82 & 56.88 & 0.00 & 47.11 \\
Solid & 39.61 & 25.98 & 6.40 & 29.93 \\
\hline
\end{tabular}


Table S5. Equipment price distribution for fast pyrolysis technology $y^{39}$

\begin{tabular}{lll}
\hline Equipment name & Cost $\mathbf{( \$ )}$ & $\begin{array}{l}\text { Contribution } \\
\mathbf{( \% )}\end{array}$ \\
\hline Bio-oil pump & 617,230 & 0.96 \\
Feed preheater & 69,455 & 0.11 \\
Gasoline cooler & 70,400 & 0.11 \\
Diesel cooler & 85,150 & 0.13 \\
Pressure swing adsorber 1 & 111,535 & 0.17 \\
Pressure swing adsorber 2 & 111,535 & 0.17 \\
Demister 1 & 117,315 & 0.18 \\
Gas-liquid separator & 130,510 & 0.20 \\
Demister 2 & 130,510 & 0.20 \\
Dry flash & 137,810 & 0.21 \\
LP Flash & 146,270 & 0.23 \\
Pressure swing adsorber 3 & 173,830 & 0.27 \\
Quencher & 192,505 & 0.30 \\
Pump & 206,305 & 0.32 \\
Cyclone separator & 228,942 & 0.35 \\
Demister 3 & 253,385 & 0.39 \\
HP-flash & 264,785 & 0.41 \\
Boiler & 300,091 & 0.46 \\
Gasoline storage tank & 309,640 & 0.48 \\
Sulfur removal & 310,215 & 0.48 \\
WGS reactor & 425,224 & 0.66 \\
Condenser & 536,000 & 0.83 \\
Diesel storage tank & 551,478 & 0.85 \\
wastewater storage & 570,159 & 0.88 \\
Distillation Column 1 & 700,005 & 1.08 \\
Distillation Column 2 & 853,490 & 1.32 \\
Generator & 960,450 & 1.49 \\
Turbine & $1,688,380$ & 2.61 \\
Reformer & $2,499,887$ & 3.87 \\
Compressor 2 & $2,765,390$ & 4.28 \\
Compressor 1 & $2,898,910$ & 4.49 \\
Compressor 3 & $3,350,415$ & 5.19 \\
Hydrotreater & $7,286,741$ & 11.28 \\
Dryer & $9,550,244$ & 14.79 \\
Hydrocracker & $12,849,618$ & 19.90 \\
Pyrolysis reactor & $13,123,210$ & 20.32 \\
\hline Total & $64,577,019$ & 100.00 \\
\hline
\end{tabular}


Table S6. Equipment price distribution for slow pyrolysis technology

\begin{tabular}{lll}
\hline Equipment name & Cost $\mathbf{( \$ )}$ & $\begin{array}{l}\text { Contribution } \\
\text { (\%) }\end{array}$ \\
\hline Bio-oil pump & 693,930 & 1.33 \\
Pump & 208,170 & 0.40 \\
Compressor 1 & 64,740 & 0.12 \\
Preheater & 66,850 & 0.13 \\
Compressor 2 & 67,660 & 0.13 \\
Pressure swing adsorber 1 & 107,450 & 0.21 \\
Demister 1 & 107,450 & 0.21 \\
Pressure swing adsorber 2 & 119,995 & 0.23 \\
LP-Flash & 122,400 & 0.23 \\
Gas-liquid separator & 125,720 & 0.24 \\
Demister 2 & 125,720 & 0.24 \\
Gasoline storage tank & 132,642 & 0.25 \\
Dry flash tank & 132,655 & 0.25 \\
Pressure swing adsorber 3 & 167,030 & 0.32 \\
Quencher & 185,210 & 0.36 \\
WGS reactor & 193,040 & 0.37 \\
HP-Flash & 210,905 & 0.40 \\
Diesel storage tank & 224,026 & 0.43 \\
Demister 3 & 244,255 & 0.47 \\
Column & 254,665 & 0.49 \\
Reformer & 358,034 & 0.69 \\
Boiler & 375,965 & 0.72 \\
Distillation Column 1 & 496,270 & 0.95 \\
wastewater storage & 552,473 & 1.06 \\
Distillation column 2 & 555,360 & 1.06 \\
Condenser & $1,025,650$ & 1.97 \\
Compressor 3 & $2,618,575$ & 5.02 \\
Hydrotreater & $3,007,832$ & 5.77 \\
Compressor 4 & $3,518,090$ & 6.74 \\
Compressor 5 & $3,942,005$ & 7.56 \\
Hydrocracker & $4,512,086$ & 8.65 \\
Turbine & $4,974,258$ & 9.54 \\
Dryer & $9,550,244$ & 18.31 \\
Pyrolysis reactor & $13,123,210$ & 25.16 \\
\hline Total & $41,164,565$ & 100.00 \\
\hline & & \\
& & \\
\hline
\end{tabular}


Table S7. Equipment price distribution for gasification technology

\begin{tabular}{lll}
\hline Equipment name & Cost $\mathbf{( \$ )}$ & $\begin{array}{l}\text { Contribution } \\
\mathbf{( \% )}\end{array}$ \\
\hline Condenser & 391,690 & 0.61 \\
Sulfur separator & 69,995 & 0.11 \\
Distillation column 2 & 971,650 & 1.51 \\
Dry flash tank & 137,810 & 0.21 \\
Compressor 1 & $2,132,910$ & 3.32 \\
F-T water knock out & 117,090 & 0.18 \\
Steam generator & 272,225 & 0.42 \\
F-T liquid cooler & $1,774,550$ & 2.76 \\
AIR-preheater & 46,577 & 0.07 \\
Column-1 & 955,645 & 1.49 \\
Feed-preheater & 44,857 & 0.07 \\
NH3-Scrubber & 139,380 & 0.22 \\
Gasoline cooler & 69,995 & 0.11 \\
Compressor-4 & $2,844,780$ & 4.43 \\
F-T liquid absorber & 117,090 & 0.18 \\
1st Sulfur Separator & 105,535 & 0.16 \\
Syngas-cooler & 581,440 & 0.91 \\
Compressor-2 & $2,091,465$ & 3.26 \\
SC water knock out & 125,890 & 0.20 \\
Compressor-air & $4,321,445$ & 6.73 \\
Wax pump & 185,625 & 0.29 \\
CO2-remover & 117,660 & 0.18 \\
Compressor-3 & $1,472,920$ & 2.29 \\
PSA & 126,030 & 0.20 \\
Lock hopper & 144,139 & 0.22 \\
Turbine & $1,394,000$ & 2.17 \\
Diesel-cooler & 86,165 & 0.13 \\
Cyclone & 85,399 & 0.13 \\
Compressor-5 & $2,708,995$ & 4.22 \\
Gasoline storage tank & 456,759 & 0.71 \\
Diesel storage tank & 515,864 & 0.80 \\
wastewater storage & 420,091 & 0.65 \\
Gasifier & $8,884,322$ & 13.84 \\
FT reactor & $5,981,756$ & 9.32 \\
Dryer & $9,548,588$ & 14.88 \\
Boiler & 375,965 & 0.59 \\
Hydrocracker & $7,585,565$ & 11.82 \\
Reformer & $1,911,564$ & 2.98 \\
Acid gas remover & $2,009,549$ & 3.13 \\
\hline
\end{tabular}




\begin{tabular}{lll} 
Claus Converter & $1,907,260$ & 2.97 \\
Hydrolysis reactor & 956,988 & 1.49 \\
& & \\
\hline Total & $64,187,222$ & 100.00 \\
\hline
\end{tabular}


Table S8. Equipment price distribution for hydrothermal liquefaction technology

\begin{tabular}{|c|c|c|}
\hline Equipment name & Cost (\$) & $\begin{array}{l}\text { Contribution } \\
(\%)\end{array}$ \\
\hline Preheater 1 & 280,615 & 0.39 \\
\hline Feed heater & 620,890 & 0.87 \\
\hline Compressor 1 & $2,489,825$ & 3.50 \\
\hline Pressure swing adsorber 1 & 131,590 & 0.18 \\
\hline Preheater 2 & 141,730 & 0.20 \\
\hline Pressure swing adsorber 2 & 126,420 & 0.18 \\
\hline Flash & 131,590 & 0.18 \\
\hline HP-Flash & 146,270 & 0.21 \\
\hline Hydrocracker & $1,340,205$ & 1.88 \\
\hline Turbine & $7,304,560$ & 10.26 \\
\hline Condenser & 280,705 & 0.39 \\
\hline Column & 982,780 & 1.38 \\
\hline Adsorption & 175,130 & 0.25 \\
\hline Heat exchanger & 117,610 & 0.17 \\
\hline Distillation column & $1,245,415$ & 1.75 \\
\hline Compressor 3 & $2,952,445$ & 4.15 \\
\hline Distillation column & 69,815 & 0.10 \\
\hline 3-phase-separator & 190,315 & 0.27 \\
\hline Distillation Column & 73,050 & 0.10 \\
\hline NH3 scrubber & 148,860 & 0.21 \\
\hline F-p-ex & 64,855 & 0.09 \\
\hline Hc-2-fl & 131,590 & 0.18 \\
\hline Cooler 1 & 520,905 & 0.73 \\
\hline Sulfur treatment & 190,315 & 0.27 \\
\hline Cooler 2 & 71,260 & 0.10 \\
\hline LP-Flash & 146,270 & 0.21 \\
\hline Feed pump & $1,883,210$ & 2.64 \\
\hline Compressor 4 & $2,283,120$ & 3.21 \\
\hline Preheater 3 & 141,730 & 0.20 \\
\hline Liquid gas separator & 124,415 & 0.17 \\
\hline Pressure swing adsorber 3 & 126,030 & 0.18 \\
\hline Cooler 3 & 105,945 & 0.15 \\
\hline Gasoline storage tank & 252,410 & 0.35 \\
\hline Diesel storage tank & 579,567 & 0.81 \\
\hline Wastewater storage & 105,668 & 0.15 \\
\hline Reformer & 2,962,192 & 4.16 \\
\hline HTL reactor & $17,287,229$ & 24.27 \\
\hline Hydrotreater & $5,481,468$ & 7.70 \\
\hline Hydrocracker & $8,364,620$ & 11.74 \\
\hline
\end{tabular}




\begin{tabular}{lll} 
Boiler & 443,408 & 0.62 \\
AD reactor & $11,011,074$ & 15.46 \\
& & \\
\hline Total & $71,227,102$ & 100.00 \\
\hline
\end{tabular}


Table S9. Price of the important utilities. ${ }^{15,40-41}$

\begin{tabular}{clclc}
\hline $\begin{array}{r}\text { Sr. } \\
\text { No. }\end{array}$ & Utility name & Price & Units & Reference No. \\
\hline 1 & Electricity & 5.4 & cents/kWh & 40 \\
2 & Process Steam & 8.2 & \$/ton & 15 \\
3 & Cooling water & 0.31 & \$/ton & 15 \\
4 & Natural gas & 7.42 & \$/thousand cubic feet (\$/MCF) & 41 \\
5 & Wastewater & 3.3 & \$/hundred cubic feet & 15 \\
\hline
\end{tabular}

Table S10. Product market prices used for the revenue calculations. ${ }^{38,42-45}$

\begin{tabular}{lll}
\hline Product & Price & Units \\
oil & 0.33 & $\$ \mathrm{M} / \mathrm{kt}$ \\
diesel & 1.03 & $\$ \mathrm{M} / \mathrm{kt}$ \\
gasoline & 0.99 & $\$ \mathrm{M} / \mathrm{kt}$ \\
biochar & 0.10 & $\$ \mathrm{M} / \mathrm{kt}$ \\
electricity & 8.00 & $\mathrm{cents} / \mathrm{kWh}$ \\
carbon tax & 0.10 & $\$ \mathrm{M} / \mathrm{kt}$ \\
biochar & 0.20 & $\$ \mathrm{M} / \mathrm{ton}$ \\
\hline
\end{tabular}

Table S11. Revenue generation distribution for the nine cases considered.

\begin{tabular}{llllllll}
\hline Case & Oil & Diesel & Gasoline & $\begin{array}{c}\text { Biochar } \\
(\mathbf{\$ M M})\end{array}$ & Electricity & Carbon & Total \\
GA-COMB & & & & \multicolumn{2}{c}{13.46} & & 13.46 \\
FP-SELL & 22.54 & & & 3.12 & 0.98 & 0.94 & 27.58 \\
HTL-SELL & 11.56 & & & 2.36 & & & 13.92 \\
SP-CHAR & 11.03 & & & 9.19 & 2.79 & 1.83 & 24.84 \\
GA-CHP & & & & & & & 26.91 \\
SP-COMB & 11.03 & & & & 4.28 & & 15.31 \\
FP-UPGRADE & & 31.11 & 10.82 & 1.56 & 0.49 & 0.47 & 44.45 \\
HTL-UPGRADE & & 25.46 & 7.33 & 2.36 & & & 35.15 \\
GA-FT & 24.68 & 17.48 & & & & 42.17 \\
\hline
\end{tabular}


Table S12. NPV calculation for FP-SELL case.

\begin{tabular}{llll}
\hline $\begin{array}{l}\text { FP-SELL } \\
\text { year }\end{array}$ & & & \\
\hline 0 & $-55,109,214$ & $-55,109,214$ & $-55,109,214$ \\
1 & $11,627,132$ & $11,073,459$ & $-44,035,755$ \\
2 & $11,627,132$ & $10,546,152$ & $-33,489,603$ \\
3 & $11,627,132$ & $10,043,954$ & $-23,445,649$ \\
4 & $11,627,132$ & $9,565,670$ & $-13,879,979$ \\
5 & $11,627,132$ & $9,110,162$ & $-4,769,817$ \\
6 & $11,627,132$ & $8,676,345$ & $3,906,528$ \\
7 & $11,627,132$ & $8,263,186$ & $12,169,714$ \\
8 & $11,627,132$ & $7,869,701$ & $20,039,415$ \\
9 & $11,627,132$ & $7,494,953$ & $27,534,368$ \\
10 & $11,627,132$ & $7,138,051$ & $34,672,418$ \\
11 & $11,627,132$ & $6,798,143$ & $41,470,562$ \\
12 & $11,627,132$ & $6,474,422$ & $47,944,984$ \\
13 & $11,627,132$ & $6,166,116$ & $54,111,101$ \\
14 & $11,627,132$ & $5,872,492$ & $59,983,592$ \\
15 & $11,627,132$ & $5,592,849$ & $65,576,442$ \\
16 & $11,627,132$ & $5,326,523$ & $70,902,965$ \\
17 & $11,627,132$ & $5,072,879$ & $75,975,844$ \\
18 & $11,627,132$ & $4,831,314$ & $80,807,158$ \\
19 & $11,627,132$ & $4,601,251$ & $85,408,409$ \\
20 & $11,627,132$ & $4,382,144$ & $89,790,553$ \\
& & & 89,79 \\
\hline
\end{tabular}


Table S13. NPV calculation for SP-CHAR case.

\begin{tabular}{llll}
\hline $\begin{array}{l}\text { SP-CHAR } \\
\text { year }\end{array}$ & & & \\
\hline 0 & $-38,871,805$ & $-38,871,805$ & $-38,871,805$ \\
1 & $16,785,210$ & $15,985,914$ & $-22,885,891$ \\
2 & $16,785,210$ & $15,224,680$ & $-7,661,211$ \\
3 & $16,785,210$ & $14,499,695$ & $6,838,485$ \\
4 & $16,785,210$ & $13,809,234$ & $20,647,718$ \\
5 & $16,785,210$ & $13,151,651$ & $33,799,370$ \\
6 & $16,785,210$ & $12,525,382$ & $46,324,752$ \\
7 & $16,785,210$ & $11,928,935$ & $58,253,687$ \\
8 & $16,785,210$ & $11,360,891$ & $69,614,578$ \\
9 & $16,785,210$ & $10,819,896$ & $80,434,474$ \\
10 & $16,785,210$ & $10,304,663$ & $90,739,137$ \\
11 & $16,785,210$ & $9,813,965$ & $100,553,101$ \\
12 & $16,785,210$ & $9,346,633$ & $109,899,734$ \\
13 & $16,785,210$ & $8,901,555$ & $118,801,289$ \\
14 & $16,785,210$ & $8,477,672$ & $127,278,961$ \\
15 & $16,785,210$ & $8,073,973$ & $135,352,934$ \\
16 & $16,785,210$ & $7,689,498$ & $143,042,432$ \\
17 & $16,785,210$ & $7,323,331$ & $150,365,764$ \\
18 & $16,785,210$ & $6,974,601$ & $157,340,365$ \\
19 & $16,785,210$ & $6,642,478$ & $163,982,843$ \\
20 & $16,785,210$ & $6,326,169$ & $170,309,012$ \\
& & & 170.31 \\
\hline
\end{tabular}


Table S14. NPV calculation for SP-COMB case.

\begin{tabular}{llll}
\hline $\begin{array}{l}\text { SP-COMB } \\
\text { year }\end{array}$ & & & \\
\hline 0 & $-41,258,986$ & $-41,258,986$ & $-41,258,986$ \\
1 & $4,121,929$ & $3,925,647$ & $-37,333,339$ \\
2 & $4,121,929$ & $3,738,711$ & $-33,594,627$ \\
3 & $4,121,929$ & $3,560,678$ & $-30,033,950$ \\
4 & $4,121,929$ & $3,391,121$ & $-26,642,828$ \\
5 & $4,121,929$ & $3,229,639$ & $-23,413,189$ \\
6 & $4,121,929$ & $3,075,847$ & $-20,337,342$ \\
7 & $4,121,929$ & $2,929,378$ & $-17,407,963$ \\
8 & $4,121,929$ & $2,789,884$ & $-14,618,079$ \\
9 & $4,121,929$ & $2,657,032$ & $-11,961,047$ \\
10 & $4,121,929$ & $2,530,507$ & $-9,430,540$ \\
11 & $4,121,929$ & $2,410,007$ & $-7,020,533$ \\
12 & $4,121,929$ & $2,295,244$ & $-4,725,289$ \\
13 & $4,121,929$ & $2,185,947$ & $-2,539,342$ \\
14 & $4,121,929$ & $2,081,854$ & $-457,487$ \\
15 & $4,121,929$ & $1,982,718$ & $1,525,231$ \\
16 & $4,121,929$ & $1,888,303$ & $3,413,535$ \\
17 & $4,121,929$ & $1,798,384$ & $5,211,919$ \\
18 & $4,121,929$ & $1,712,747$ & $6,924,666$ \\
19 & $4,121,929$ & $1,631,187$ & $8,555,853$ \\
20 & $4,121,929$ & $1,553,512$ & $10,109,365$ \\
& & & 10.11 \\
\hline
\end{tabular}


Table S15. GWP 100-year factors of some of the chemicals involved in the simulations. The values are directly obtained from the IPCC report on Climate Change in $2013^{46}$

\begin{tabular}{cccc}
\hline & Acronym, Common & Chemical & GWP 100-year $\left(\mathrm{kg} \mathrm{CO}_{2}-\right.$ \\
Sr. No. & Name & Formula & Eq) \\
\hline 1 & Carbon dioxide & $\mathrm{CO}_{2}$ & 1 \\
2 & Methane & $\mathrm{CH}_{4}$ & 28 \\
3 & Nitrous oxide & $\mathrm{N}_{2} \mathrm{O}$ & 265 \\
4 & Nitrous oxides & $\mathrm{NOx}$ & -8.2 \\
5 & Carbon monoxide & $\mathrm{CO}$ & 1.8 \\
6 & Sulphur dioxide & $\mathrm{SO}_{2}$ & -38.4 \\
\hline
\end{tabular}

Table S16. Net present value (NPV) and greenhouse gas (GHG) inventory results for the nine considered cases.

\begin{tabular}{lrr}
\hline Case & GHG (kg C $\mathbf{C O}_{2}$-eq) & NPV (\$MM) \\
GA-FT & 339.00 & 234.33 \\
GA-CHP & 378.35 & 72.15 \\
GA-COMB & 400.00 & 25.02 \\
SP-COMB & 270.00 & 10.00 \\
SP-CHAR & 279.00 & 170.00 \\
FP-SELL & 217.00 & 89.79 \\
FP-UPGRADE & 361.50 & 314.49 \\
HTL-SELL & 339.40 & 28.40 \\
HTL-UPGRADE & 494.50 & 196.27 \\
\hline
\end{tabular}




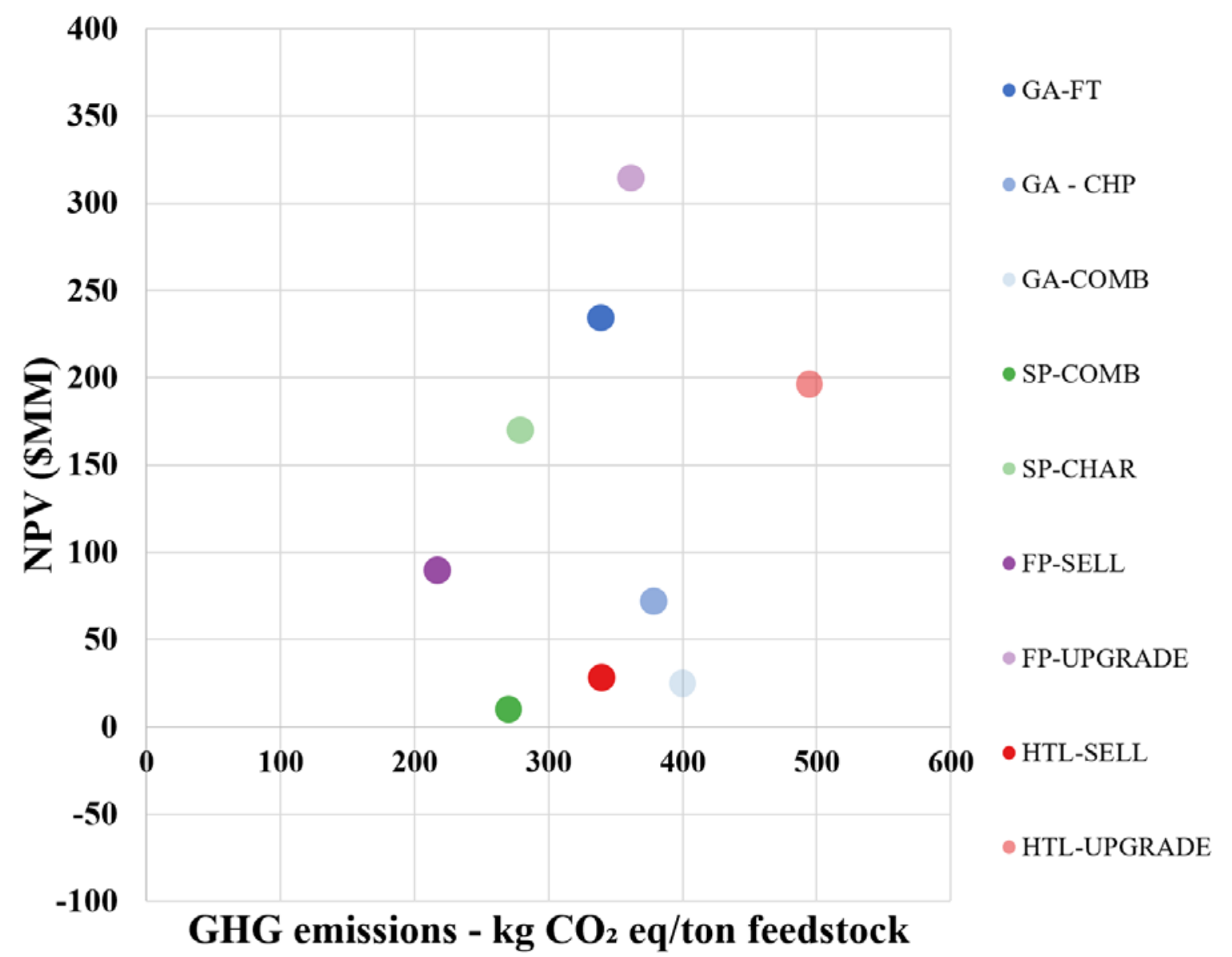

Figure S3. Trade-off between economic and environmental performance illustrated through the plot of NPVs (\$MM) against greenhouse gas emissions (GHG) for the nine cases.

Table S17. Syngas production potential through slow pyrolysis for the 14 CAFOs and the corresponding power that can be generated. ${ }^{16}$

\begin{tabular}{lrrrr}
\hline Sr. No. & $\begin{array}{r}\text { Syngas } \\
(\mathrm{kg} / \mathrm{hr})\end{array}$ & $\begin{array}{r}\text { Energy } \\
(\mathrm{MW})\end{array}$ & $\begin{array}{r}\text { Electricity } \\
(\mathrm{MW})\end{array}$ & $\begin{array}{r}\text { Heat } \\
(\mathrm{MJ} / \mathrm{hr})\end{array}$ \\
1 & $1,234.66$ & 3.92 & 1.18 & $7,053.28$ \\
2 & 811.00 & 2.57 & 0.77 & $4,633.05$ \\
3 & $1,843.45$ & 5.85 & 1.76 & $10,531.14$ \\
4 & 251.90 & 0.80 & 0.24 & $1,439.04$ \\
5 & 214.61 & 0.68 & 0.20 & $1,226.02$ \\
6 & 463.19 & 1.47 & 0.44 & $2,646.08$ \\
7 & 37.66 & 0.12 & 0.04 & 215.16 \\
8 & 121.55 & 0.39 & 0.12 & 694.40 \\
9 & 195.19 & 0.62 & 0.19 & $1,115.10$ \\
\hline
\end{tabular}




\begin{tabular}{lrlrr}
\hline 10 & 263.60 & 0.84 & 0.25 & $1,505.86$ \\
11 & 66.11 & 0.21 & 0.06 & 377.67 \\
12 & 387.58 & 1.23 & 0.37 & $2,214.16$ \\
13 & 60.12 & 0.19 & 0.06 & 343.46 \\
14 & $2,254.19$ & 7.15 & 2.15 & $12,877.60$ \\
& & & & \\
Min gas & $1,050.28$ & & 1.00 & $6,000.00$ \\
\hline
\end{tabular}

Table S18. Syngas production potential through fast pyrolysis for the 14 CAFOs and the corresponding power that can be generated. ${ }^{9}$

\begin{tabular}{|c|c|c|c|c|}
\hline Sr. No. & $\begin{array}{l}\text { Syngas } \\
(\mathrm{kg} / \mathrm{hr})\end{array}$ & $\begin{array}{r}\text { Energy } \\
\text { (MW) }\end{array}$ & $\begin{array}{r}\text { Electricity } \\
(\mathrm{MW})\end{array}$ & $\begin{array}{r}\text { Heat } \\
(\mathrm{MJ} / \mathrm{hr})\end{array}$ \\
\hline 1 & 878.81 & 1.75 & 0.52 & $3,141.57$ \\
\hline 2 & 577.26 & 1.15 & 0.34 & $2,063.59$ \\
\hline 3 & $1,312.14$ & 2.61 & 0.78 & 4,690.63 \\
\hline 4 & 179.30 & 0.36 & 0.11 & 640.96 \\
\hline 5 & 152.76 & 0.30 & 0.09 & 546.08 \\
\hline 6 & 329.69 & 0.65 & 0.20 & $1,178.58$ \\
\hline 7 & 26.81 & 0.05 & 0.02 & 95.83 \\
\hline 8 & 86.52 & 0.17 & 0.05 & 309.29 \\
\hline 9 & 138.94 & 0.28 & 0.08 & 496.67 \\
\hline 10 & 187.62 & 0.37 & 0.11 & 670.72 \\
\hline 11 & 47.06 & 0.09 & 0.03 & 168.22 \\
\hline 12 & 275.88 & 0.55 & 0.16 & 986.20 \\
\hline 13 & 42.79 & 0.08 & 0.03 & 152.98 \\
\hline 14 & $1,604.50$ & 3.19 & 0.96 & $5,735.76$ \\
\hline Min gas & $1,678.42$ & & 1.00 & $6,000.00$ \\
\hline
\end{tabular}

3.2. Sensitivity analysis results. Through the sensitivity analysis for both the slow pyrolysis and fast pyrolysis cases, parameters which would have a major impact on the NPV values for each case are identified (Figure S4). For the fast pyrolysis case (FP-UPGRADE), the plant capacity is the dominating factor with a negative NPV of -\$32MM (decrease of 110\%) on moving from the existing capacity (175 kton/year) to the lowest capacity ( $25 \mathrm{kton} / \mathrm{year}$, as determined in the spatial analysis for NYS later). This could be explained by the fact that the building of an upgrading 
facility dedicated solely to process bio-oil would be a very expensive proposition if the scale is not large enough. Both bio-oil yield (ranging from $-49.0 \%$ to $+38.2 \%)$ and diesel price $(-47.4 \%$ to $+30.8 \%$ ) are other influential parameters, thus further establishing the importance of optimizing the utilization and processing steps of bio-oil for fast pyrolysis. Other parameters such as discount rate, equipment cost and carbon credits have a smaller impact on the NPV, which is positive even for the lower bounds of all parameter values except for plant capacity.

For slow pyrolysis on the other hand, the biochar price (with a base value of $\$ 100 /$ ton) is found to be capable of dictating the overall economic performance of the plant, with a meagre NPV of $\$ 12 \mathrm{MM}$ at $\$ 0 /$ ton biochar, and a substantial NPV of $\$ 298 \mathrm{MM}$ at $\$ 500 /$ ton biochar. Towards the higher end of the biochar price spectrum, it is found that the slow pyrolysis system could compete with the fast pyrolysis system in terms of NPV and even surpass it if in combination with a high carbon credit value ( $+73 \%$ for $\$ 500 / \mathrm{CO}_{2}$-eq). Additionally, there are other environmental benefits that slow pyrolysis possesses which have not been monetized or incentivized yet. Thus, the biochar and carbon credit prices in the future could play a huge role in dictating which of the two technologies would be deployed at a larger scale.

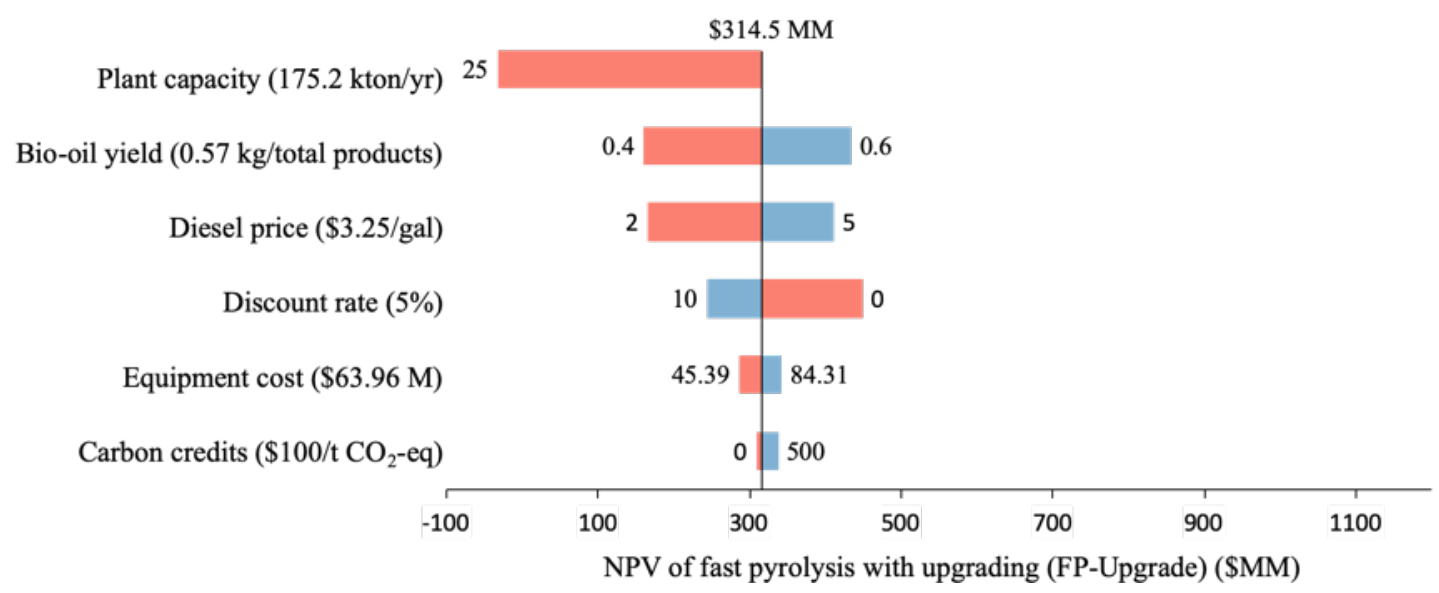




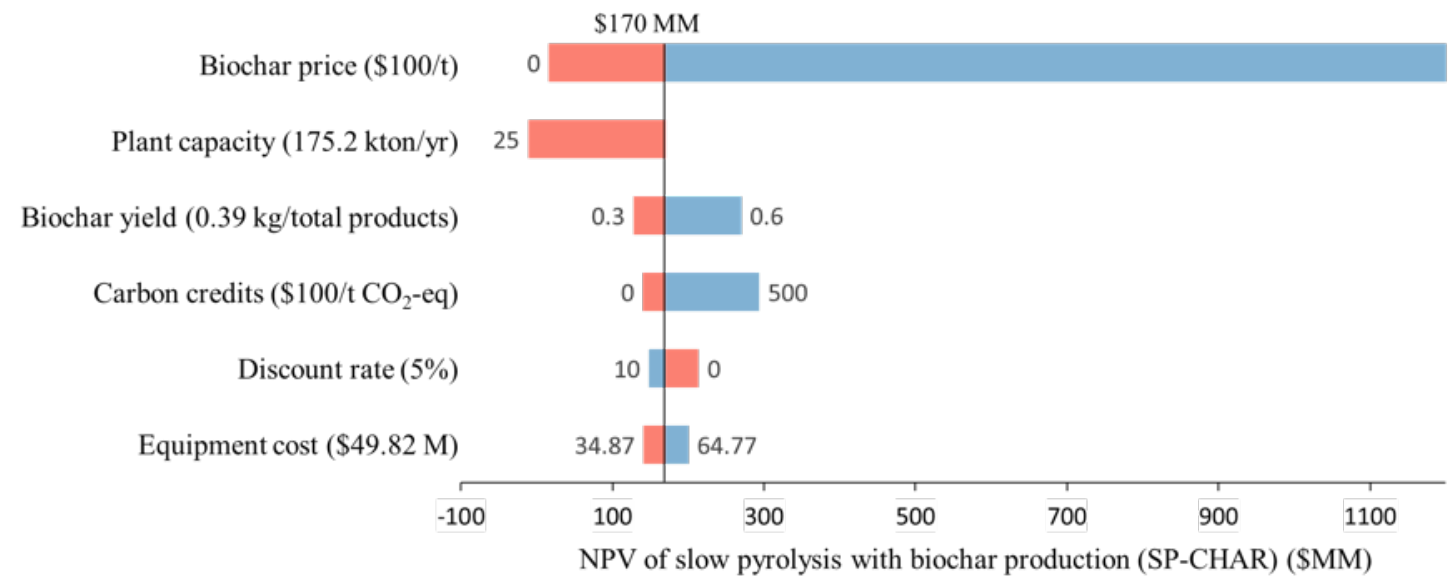

Figure S4. Sensitivity analysis results for the FP-UPGRADE (above) and SP-CHAR (below) cases. The text on the left refers to the parameters being varied, and their base values are placed in parenthesis. The labels at the end of each bar represent the extreme values of the parameter considered. The single number near the central axis for both the cases refers to the base value of the NPV (\$315MM for the fast pyrolysis case, and \$170MM for the slow pyrolysis case).

\section{SPATIAL ANALYSIS RESULTS}

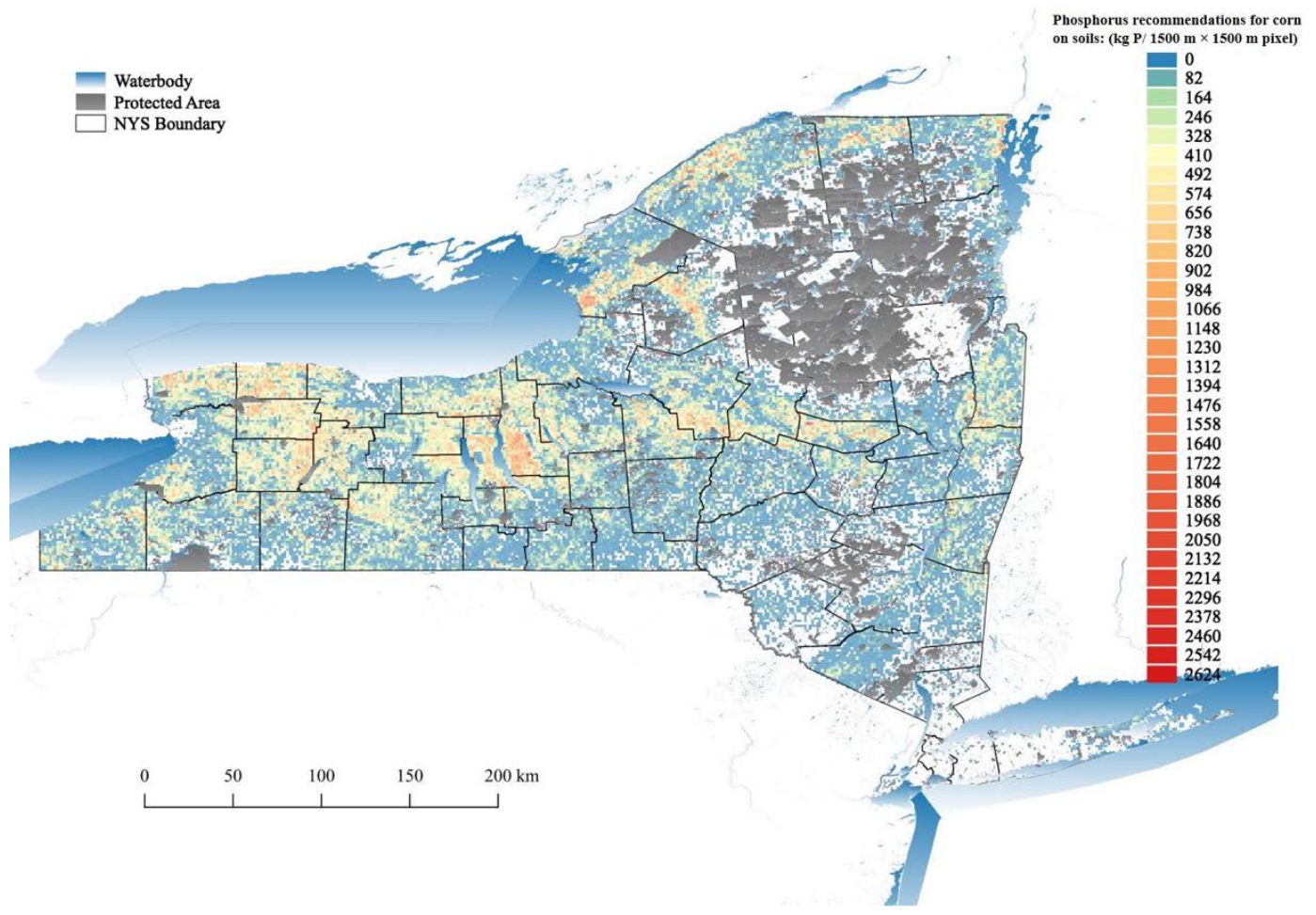


Figure S5. Spatial distribution of phosphorus in New York State (NYS).
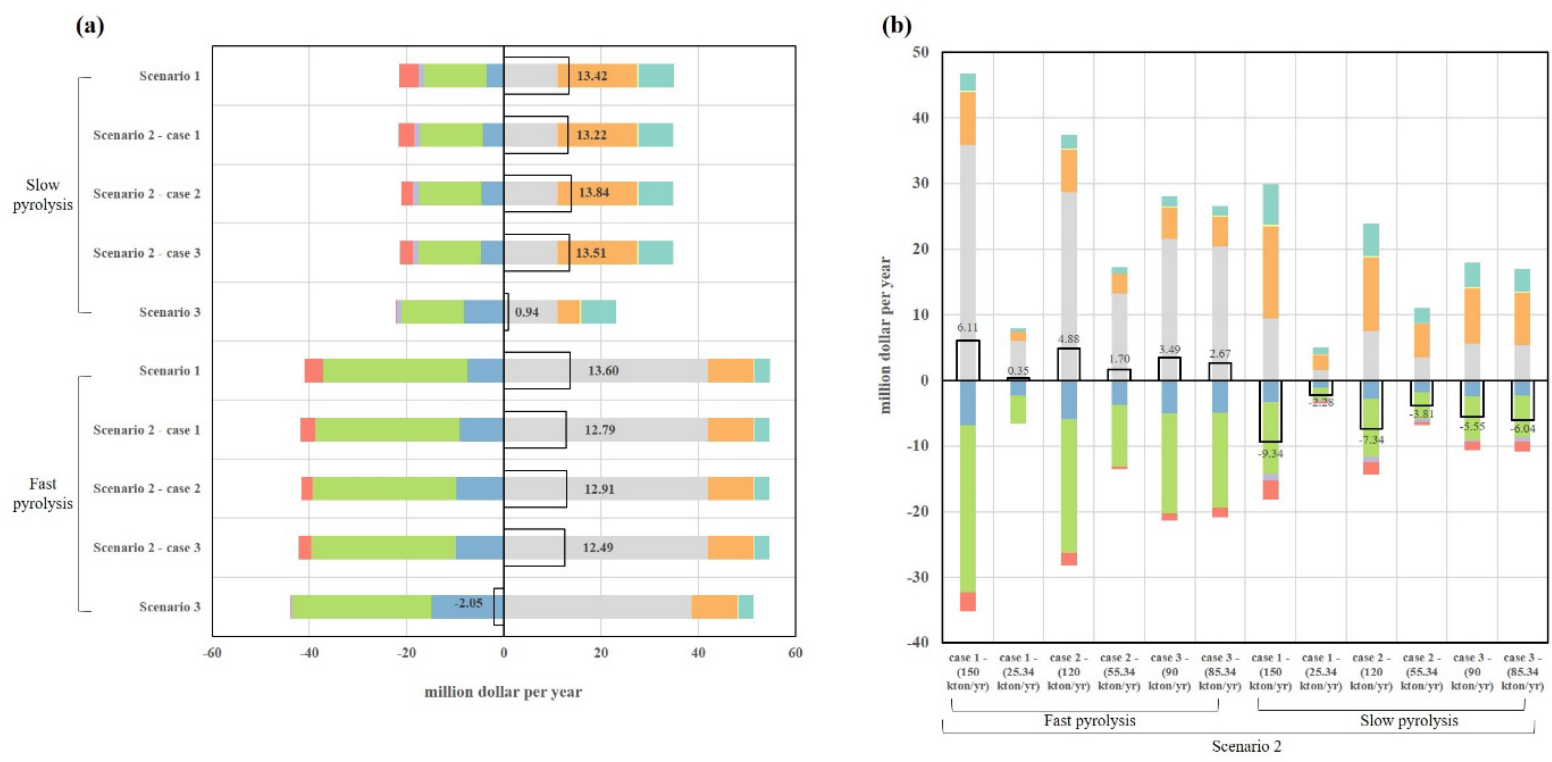

Figure S6. (a) Annualized economic breakdown of all scenarios for slow and fast pyrolysis in NYS (b) Annualized economic breakdown of all cases of Scenario 2 for slow and fast pyrolysis in NYS.

\subsection{Spatial distribution of soil phosphorus contents does not constrain application:}

Since most of the P present in the feedstock is assumed to be transferred into the biochar during the pyrolysis processes, due consideration must be given to the changes in the soil $\mathrm{P}$ contents to avoid overfertilization and consequent nutrient run-off after biochar application. The best available data for the spatial distribution of P in NYS is based on the county level data instead of pixel level or farm level (Figure S5). Even with that approximation, data for certain counties such as Warren and Hamilton cannot be obtained. Thus, we combined the county-wise recommended P application data for soils on which corn is growing with the pixel $(1500 \times 1500$ meter resolution) level corn cropland data in NYS. Then, we identified and plotted the corn cropland pixels where it is recommended to apply P (i.e. for those pixels where neither existence of corn cropland $=0$ nor recommended $\mathrm{P}$ application value $=0$ ) as shown in Figure S5. This provides a profile of biochar applicability on corn croplands in NYS. We then calculate the transportation of biochar based on these assumptions and then create Figures 4-6. Furthermore, the maximum possible P that can be found in the biochar from the entire state's CAFO poultry litter is $2.1 \mathrm{kton} / \mathrm{year}$ which is much 
lower than the recommended $5.7 \mathrm{kton} /$ year for NYS. Hence, when looking at the entire state as a whole, over-application of $\mathrm{P}$ can be avoided with appropriate transportation within the state.

\section{ADDITIONAL INFORMATION AND DATA}

Table S19. Details and poultry numbers in each CAFO for NYS. ${ }^{36,47}$

\begin{tabular}{|c|c|c|c|c|c|c|}
\hline Facility Name & County & Broilers & Layers & $\begin{array}{l}\text { Manure } \\
\text { (MT/yr) }\end{array}$ & Latitude & Longitude \\
\hline C.A.C.L. Properties Llc. & Clinton & 0.00 & $40,000.00$ & $1,285.00$ & 43.00 & -78.58 \\
\hline Giroux'S Poultry Farm & Clinton & 0.00 & $1,500,000.00$ & $48,180.00$ & 42.02 & -77.71 \\
\hline $\begin{array}{l}\text { Kreher'S Farm Fresh Eggs, } \\
\text { Llc }\end{array}$ & Erie & $251,531.00$ & $640,070.00$ & $26,389.00$ & 43.24 & -76.80 \\
\hline Sunrise Farms Inc & Greene & 0.00 & $44,000.00$ & $1,413.00$ & 43.03 & -76.39 \\
\hline Hudson Egg Farms, Llc & Onondaga & 0.00 & $167,616.00$ & $5,384.00$ & 42.89 & -76.04 \\
\hline Smith Quality Eggs, Llc. & Onondaga & $51,000.00$ & $106,000.00$ & $4,587.00$ & 41.76 & -74.94 \\
\hline Ace Farm & Orange & $56,000.00$ & $135,000.00$ & $5,634.00$ & 41.75 & -74.77 \\
\hline $\begin{array}{l}\text { Tomas Poultry Farm Of } \\
\text { Schuylevil }\end{array}$ & Saratoga & $69,206.00$ & $207,957.00$ & $8,284.00$ & 41.76 & -74.74 \\
\hline Whitesville Farms, Llc & Steuben & $150,595.00$ & $431,004.00$ & $17,334.00$ & 41.71 & -74.76 \\
\hline Harold Brey \& Sons Inc & Sullivan & $180,000.00$ & 0.00 & $4,172.00$ & 41.33 & -74.15 \\
\hline Labelle Farm & Sullivan & $80,000.00$ & $240,000.00$ & $9,900.00$ & 42.22 & -73.97 \\
\hline Hvfg, Llc & Sullivan & 0.00 & 0.00 & $2,598.00$ & 43.16 & -73.61 \\
\hline Bella Poultry Inc. & Sullivan & 0.00 & 0.00 & 805.00 & 44.89 & -73.44 \\
\hline Wayne County Eggs, Llc & Wayne & $213,357.00$ & $1,072,724.00$ & $39,401.00$ & 44.86 & -73.43 \\
\hline
\end{tabular}


Table S20. Distribution of poultry numbers in NYS based on counties. ${ }^{47}$

\begin{tabular}{lrrrrr}
\hline County & Layers & Broilers & Turkeys & Latitude & Longitude \\
Albany & $3,557.00$ & 406.00 & $\mathrm{D}$ & 42.60 & -73.97 \\
Allegany & $18,772.00$ & 344.00 & 98.00 & 42.26 & -78.03 \\
Bronx & $\mathrm{NA}$ & $\mathrm{NA}$ & $\mathrm{NA}$ & 40.85 & -73.85 \\
Broome & $3,025.00$ & 296.00 & 126.00 & 42.16 & -75.82 \\
Cattaraugus & $3,539.00$ & $1,058.00$ & 153.00 & 42.25 & -78.68 \\
Cayuga & $34,492.00$ & 462.00 & 118.00 & 43.01 & -76.57 \\
Chautauqua & $5,887.00$ & $2,725.00$ & 665.00 & 42.30 & -79.41 \\
Chemung & $1,114.00$ & $\mathrm{D}$ & 256.00 & 42.14 & -76.76 \\
Chenango & $5,453.00$ & $\mathrm{D}$ & 424.00 & 42.49 & -75.61 \\
Clinton & $\mathrm{D}$ & $1,040.00$ & 48.00 & 44.75 & -73.68 \\
Columbia & $9,359.00$ & $2,558.00$ & 393.00 & 42.25 & -73.63 \\
Cortland & $1,710.00$ & $\mathrm{D}$ & $\mathrm{D}$ & 42.60 & -76.07 \\
Delaware & $5,905.00$ & $1,254.00$ & 562.00 & 42.20 & -74.97 \\
Dutchess & $4,542.00$ & $14,301.00$ & 57.00 & 41.77 & -73.74 \\
Erie & $\mathrm{D}$ & $11,660.00$ & $\mathrm{D}$ & 42.76 & -78.78 \\
Essex & $6,317.00$ & $3,814.00$ & 583.00 & 44.12 & -73.77 \\
Franklin & $84,234.00$ & 636.00 & 78.00 & 44.59 & -74.30 \\
Fulton & $5,228.00$ & 126.00 & 21.00 & 43.11 & -74.42 \\
Genesee & $\mathrm{D}$ & 466.00 & 258.00 & 43.00 & -78.19 \\
Greene & $\mathrm{D}$ & 206.00 & 83.00 & 42.28 & -74.12 \\
Hamilton & $\mathrm{NA}$ & $\mathrm{NA}$ & $\mathrm{NA}$ & 43.66 & -74.50 \\
Herkimer & $10,079.00$ & $4,372.00$ & 77.00 & 43.42 & -74.96 \\
Jefferson & $\mathrm{D}$ & 870.00 & 149.00 & 44.00 & -76.05 \\
Kings & 28.00 & $\mathrm{NA}$ & $\mathrm{NA}$ & 40.63 & -73.95 \\
Lewis & $2,772.00$ & 360.00 & 46.00 & 43.78 & -75.45 \\
Livingston & $2,577.00$ & 282.00 & 39.00 & 42.73 & -77.78 \\
Madison & $2,902.00$ & $1,288.00$ & 332.00 & 42.91 & -75.67 \\
Monroe & $1,902.00$ & 723.00 & 189.00 & 43.31 & -77.68 \\
Montgomery & $5,034.00$ & 815.00 & 252.00 & 42.90 & -74.44 \\
Nassau & 60.00 & $\mathrm{NA}$ & $\mathrm{NA}$ & 40.73 & -73.59 \\
New York & 36.00 & $\mathrm{NA}$ & $\mathrm{NA}$ & 40.77 & -73.97 \\
Niagara & $4,067.00$ & $1,881.00$ & 280.00 & 43.34 & -78.77 \\
Oneida & $5,744.00$ & $1,142.00$ & 131.00 & 43.24 & -75.44 \\
Onondaga & $703,150.00$ & 751.00 & $\mathrm{D}$ & 43.01 & -76.19 \\
Ontario & $40,723.00$ & 643.00 & 116.00 & 42.85 & -77.30 \\
Orange & $\mathrm{D}$ & 619.00 & 87.00 & 41.40 & -74.31 \\
Orleans & $2,832.00$ & 310.00 & $\mathrm{D}$ & 43.38 & -78.23 \\
\hline & & & & &
\end{tabular}




\begin{tabular}{lrrrrr}
\hline Oswego & $2,607.00$ & 832.00 & 274.00 & 43.46 & -76.21 \\
Otsego & $8,161.00$ & $1,005.00$ & 122.00 & 42.63 & -75.03 \\
Putnam & 899.00 & 133.00 & 12.00 & 41.43 & -73.75 \\
Queens & 550.00 & $\mathrm{NA}$ & $\mathrm{NA}$ & 40.66 & -73.84 \\
Rensselaer & $6,252.00$ & $1,620.00$ & 354.00 & 42.71 & -73.51 \\
Richmond & $\mathrm{NA}$ & $\mathrm{NA}$ & $\mathrm{NA}$ & 40.56 & -74.14 \\
Rockland & 359.00 & $\mathrm{NA}$ & $\mathrm{NA}$ & 41.15 & -74.02 \\
St Lawrence & $7,038.00$ & $2,874.00$ & 726.00 & 44.50 & -75.07 \\
Saratoga & $\mathrm{D}$ & 697.00 & 243.00 & 43.11 & -73.86 \\
Schenectady & 665.00 & 100.00 & $\mathrm{D}$ & 42.82 & -74.06 \\
Schoharie & $5,965.00$ & $2,080.00$ & 247.00 & 42.59 & -74.44 \\
Schuyler & $15,219.00$ & 338.00 & $\mathrm{D}$ & 42.39 & -76.88 \\
Seneca & $68,095.00$ & $\mathrm{D}$ & $1,259.00$ & 42.78 & -76.82 \\
Steuben & $\mathrm{D}$ & $1,034.00$ & 213.00 & 42.27 & -77.38 \\
Suffolk & $8,065.00$ & $\mathrm{D}$ & $5,190.00$ & 40.94 & -72.69 \\
Sullivan & $\mathrm{D}$ & $\mathrm{D}$ & $\mathrm{D}$ & 41.72 & -74.77 \\
Tioga & $2,317.00$ & 405.00 & 211.00 & 42.17 & -76.31 \\
Tompkins & $2,724.00$ & 305.00 & 59.00 & 42.45 & -76.47 \\
Ulster & $4,855.00$ & $1,395.00$ & 349.00 & 41.89 & -74.26 \\
Warren & 782.00 & 70.00 & $\mathrm{D}$ & 43.56 & -73.85 \\
Washington & $7,395.00$ & $\mathrm{D}$ & $1,146.00$ & 43.31 & -73.43 \\
Wayne & $\mathrm{D}$ & 810.00 & 57.00 & 43.33 & -77.05 \\
Westchester & $4,134.00$ & 440.00 & $\mathrm{D}$ & 41.15 & -73.75 \\
Wyoming & $2,485.00$ & 267.00 & 125.00 & 42.70 & -78.22 \\
Yates & $82,637.00$ & $1,371.00$ & 137.00 & 42.63 & -77.11 \\
\hline & & & & &
\end{tabular}


Table S21. Parameters and their values, units and meaning for the spatial analysis.

\begin{tabular}{|c|c|c|}
\hline Parameter & Value & Unit \\
\hline discount rate & 0.050 & \\
\hline project lifetime & 20.000 & years \\
\hline ratio of carbon content of biochar for SP & 0.397 & \\
\hline ratio of carbon content of biochar for FP & 0.300 & \\
\hline ratio of biochar production for SP & 0.262 & \\
\hline ratio of biochar production for FP & 0.178 & \\
\hline ratio of bio-crude production for SP & 0.191 & \\
\hline ratio of bio-crude production for FP & 0.390 & \\
\hline ratio of gas production for SP & 0.209 & \\
\hline ratio of gas production for $\mathrm{FP}$ & 0.118 & \\
\hline ratio of diesel production from FP oil & 0.440 & \\
\hline ratio of gasoline production from FP oil & 0.160 & \\
\hline OM cost for SP with CHP (175.2 kt/yr) & 21.336 & \\
\hline OM cost for SP with combustion (175.2 kt/yr) & 21.200 & \\
\hline OM cost for FP with CHP and upgrading (175.2 kt/yr) & 29.692 & \\
\hline OM cost for FP with CHP and no upgrading (175.2 kt/yr) & 23.107 & \\
\hline OM cost for FP with combustion and upgrading (175.2 kt/yr) & 29.623 & \\
\hline OM cost for FP with combustion and no upgrading (175.2 kt/yr) & 23.039 & \\
\hline CC cost for SP with CHP (175.2 kt/yr) & 63.793 & \\
\hline CC cost for SP with combustion (175.2 kt/yr) & 61.127 & \\
\hline CC cost for FP with CHP and upgrading (175.2 kt/yr) & 93.182 & \\
\hline CC cost for FP with CHP and no upgrading (175.2 kt/yr) & 59.195 & \\
\hline CC cost for FP with combustion and upgrading (175.2 kt/yr) & 91.849 & \\
\hline CC cost for FP with combustion and no upgrading (175.2 kt/yr) & 57.862 & \\
\hline SP biochar price & 0.300 & $\$ \mathrm{M} / \mathrm{kt}$ \\
\hline $\begin{array}{l}\text { FP biochar price } \\
\text { bio-crude price for both SP and FP (derived from } \$ 45 / \text { barrel and } 7.33 \text { barrel/t bio- } \\
\text { crude) }\end{array}$ & 0.300 & $\begin{array}{l}\$ \mathrm{M} / \mathrm{kt} \\
\$ \mathrm{M} / \mathrm{kt}\end{array}$ \\
\hline diesel price (derived from 325.9 cent/gal and 6.943 lb/gal diesel) & 1.035 & $\$ \mathrm{M} / \mathrm{kt}$ \\
\hline gasoline price (derived from 279.0 cent/gal and 2.79 lb/gal diesel) & 0.989 & $\$ \mathrm{M} / \mathrm{kt}$ \\
\hline the maximum ratio that can be accepted to each refinery & 0.010 & \\
\hline Carbon Tax & 100.000 & $\$ / \mathrm{tCO}_{2} \mathrm{eq}$ \\
\hline Min gas flow for CHP - SP (for 500 MWe) & 4.600 & kton/yr \\
\hline Min gas flow for CHP - FP (for $250 \mathrm{MWe}$ ) & 3.676 & $\mathrm{kton} / \mathrm{yr}$ \\
\hline CHP efficiency & 0.800 & \\
\hline CHP electricity efficiency & 0.300 & \\
\hline CHP heat efficiency & 0.500 & \\
\hline
\end{tabular}


Table S22. List of considered existing refinery equipment that is capable of processing certain amounts of produced bio-oil. ${ }^{34}$ Total operable capacity was considered while calculating the fraction of bio-oil that could potentially be accepted in each refinery. Ultimately, only the last two refineries listed in the table were considered in the analysis.

\begin{tabular}{|c|c|c|c|}
\hline Company_name & Site & Product & $\begin{array}{l}\text { Quantity } \\
\text { (barrels/stream day) }\end{array}$ \\
\hline American refining group inc & Bradford & Asphalt \& road oil & 65 \\
\hline American refining group inc & Bradford & Asphalt \& road oil & 65 \\
\hline American refining group inc & Bradford & Cat reforming: high pressure & 1800 \\
\hline American refining group inc & Bradford & Cat reforming: high pressure & 2200 \\
\hline American refining group inc & Bradford & Cat reforming: high pressure & 2200 \\
\hline American refining group inc & Bradford & Desulfurization, naphtha/reformer feed & 3600 \\
\hline American refining group inc & Bradford & Desulfurization, naphtha/reformer feed & 3600 \\
\hline American refining group inc & Bradford & Lubricants & 2945 \\
\hline American refining group inc & Bradford & Lubricants & 2945 \\
\hline American refining group inc & Bradford & Operating capacity & 11000 \\
\hline American refining group inc & Bradford & Operating capacity & 11800 \\
\hline American refining group inc & Bradford & Total oper cap (projected, next year) & 11800 \\
\hline American refining group inc & Bradford & Total operable capacity & 11000 \\
\hline American refining group inc & Bradford & Total operable capacity & 11800 \\
\hline Monroe energy llc & Trainer & Alkylates & 12000 \\
\hline Monroe energy llc & Trainer & Alkylates & 12000 \\
\hline Monroe energy llc & Trainer & Cat cracking: fresh feed & 51500 \\
\hline Monroe energy llc & Trainer & Cat cracking: fresh feed & 53000 \\
\hline Monroe energy llc & Trainer & Cat cracking: fresh feed & 53000 \\
\hline Monroe energy llc & Trainer & Cat hydrocracking, distillate & 21500 \\
\hline Monroe energy llc & Trainer & Cat hydrocracking, distillate & 23000 \\
\hline Monroe energy llc & Trainer & Cat hydrocracking, distillate & 23000 \\
\hline Monroe energy llc & Trainer & Cat reforming: low pressure & 45000 \\
\hline Monroe energy llc & Trainer & Cat reforming: low pressure & 50000 \\
\hline Monroe energy llc & Trainer & Cat reforming: low pressure & 50000 \\
\hline Monroe energy llc & Trainer & Desulfurization, diesel fuel & 53300 \\
\hline Monroe energy llc & Trainer & Desulfurization, diesel fuel & 53300 \\
\hline Monroe energy llc & Trainer & Desulfurization, gasoline & 34000 \\
\hline Monroe energy llc & Trainer & Desulfurization, gasoline & 34000 \\
\hline Monroe energy llc & Trainer & Desulfurization, kerosene and jet & 23300 \\
\hline Monroe energy llc & Trainer & Desulfurization, kerosene and jet & 23300 \\
\hline Monroe energy llc & Trainer & Desulfurization, naphtha/reformer feed & 80000 \\
\hline Monroe energy llc & Trainer & Desulfurization, naphtha/reformer feed & 80000 \\
\hline Monroe energy llc & Trainer & Operating capacity & 190000 \\
\hline Monroe energy llc & Trainer & Operating capacity & 208000 \\
\hline
\end{tabular}




\begin{tabular}{|c|c|c|c|}
\hline Monroe energy llc & Trainer & Sulfur (short tons/day) & 90 \\
\hline Monroe energy llc & Trainer & Sulfur (short tons/day) & 90 \\
\hline Monroe energy llc & Trainer & Total oper cap (projected, next year) & 208000 \\
\hline Monroe energy llc & Trainer & Total operable capacity & 190000 \\
\hline Monroe energy llc & Trainer & Total operable capacity & 208000 \\
\hline Monroe energy llc & Trainer & Vacuum distillation & 73000 \\
\hline Monroe energy llc & Trainer & Vacuum distillation & 73000 \\
\hline Paulsboro refining co llc & Paulsboro & Alkylates & 11200 \\
\hline Paulsboro refining co llc & Paulsboro & Alkylates & 11200 \\
\hline Paulsboro refining co llc & Paulsboro & Asphalt \& road oil & 21000 \\
\hline Paulsboro refining co llc & Paulsboro & Asphalt \& road oil & 21000 \\
\hline Paulsboro refining co llc & Paulsboro & Cat cracking: fresh feed & 54000 \\
\hline Paulsboro refining co llc & Paulsboro & Cat cracking: fresh feed & 55000 \\
\hline Paulsboro refining co llc & Paulsboro & Cat cracking: fresh feed & 55000 \\
\hline Paulsboro refining co llc & Paulsboro & Cat reforming: low pressure & 28500 \\
\hline Paulsboro refining co llc & Paulsboro & Cat reforming: low pressure & 32000 \\
\hline Paulsboro refining co llc & Paulsboro & Cat reforming: low pressure & 32000 \\
\hline Paulsboro refining co llc & Paulsboro & Desulfurization, diesel fuel & 46000 \\
\hline Paulsboro refining co llc & Paulsboro & Desulfurization, diesel fuel & 46000 \\
\hline Paulsboro refining co llc & Paulsboro & Desulfurization, gasoline & 37000 \\
\hline Paulsboro refining co llc & Paulsboro & Desulfurization, gasoline & 37000 \\
\hline Paulsboro refining co llc & Paulsboro & Desulfurization, kerosene and jet & 29100 \\
\hline Paulsboro refining co llc & Paulsboro & Desulfurization, kerosene and jet & 29100 \\
\hline Paulsboro refining co llc & Paulsboro & Desulfurization, naphtha/reformer feed & 32000 \\
\hline Paulsboro refining co llc & Paulsboro & Desulfurization, naphtha/reformer feed & 32000 \\
\hline Paulsboro refining co llc & Paulsboro & Hydrogen (mmcfd) & 9 \\
\hline Paulsboro refining co llc & Paulsboro & Hydrogen (mmcfd) & 9 \\
\hline Paulsboro refining co llc & Paulsboro & Lubricants & 12000 \\
\hline Paulsboro refining co llc & Paulsboro & Lubricants & 12000 \\
\hline Paulsboro refining co llc & Paulsboro & Operating capacity & 160000 \\
\hline Paulsboro refining co llc & Paulsboro & Operating capacity & 166000 \\
\hline Paulsboro refining co llc & Paulsboro & Petcoke,market & 7500 \\
\hline Paulsboro refining co llc & Paulsboro & Petcoke,market & 7500 \\
\hline Paulsboro refining co llc & Paulsboro & Sulfur (short tons/day) & 280 \\
\hline Paulsboro refining co llc & Paulsboro & Sulfur (short tons/day) & 350 \\
\hline Paulsboro refining co llc & Paulsboro & Therm cracking, delayed coking & 26500 \\
\hline Paulsboro refining co llc & Paulsboro & Therm cracking, delayed coking & 27000 \\
\hline Paulsboro refining co llc & Paulsboro & Therm cracking, delayed coking & 27000 \\
\hline Paulsboro refining co llc & Paulsboro & Total oper cap (projected, next year) & 166000 \\
\hline Paulsboro refining co llc & Paulsboro & Total operable capacity & 160000 \\
\hline Paulsboro refining co llc & Paulsboro & Total operable capacity & 166000 \\
\hline Paulsboro refining co llc & Paulsboro & Vacuum distillation & 90000 \\
\hline Paulsboro refining co llc & Paulsboro & Vacuum distillation & 90000 \\
\hline
\end{tabular}




\begin{tabular}{|c|c|c|c|}
\hline Philadelphia energy solutions & Philadelphia & Alkylates & 26500 \\
\hline Philadelphia energy solutions & Philadelphia & Alkylates & 26500 \\
\hline Philadelphia energy solutions & Philadelphia & Aromatics & 4920 \\
\hline Philadelphia energy solutions & Philadelphia & Aromatics & 4920 \\
\hline Philadelphia energy solutions & Philadelphia & Cat cracking: fresh feed & 127300 \\
\hline Philadelphia energy solutions & Philadelphia & Cat cracking: fresh feed & 137500 \\
\hline Philadelphia energy solutions & Philadelphia & Cat cracking: fresh feed & 137500 \\
\hline Philadelphia energy solutions & Philadelphia & Cat reforming: high pressure & 77400 \\
\hline Philadelphia energy solutions & Philadelphia & Cat reforming: high pressure & 86000 \\
\hline Philadelphia energy solutions & Philadelphia & Cat reforming: high pressure & 86000 \\
\hline Philadelphia energy solutions & Philadelphia & Desulfurization, gasoline & 65000 \\
\hline Philadelphia energy solutions & Philadelphia & Desulfurization, gasoline & 65000 \\
\hline Philadelphia energy solutions & Philadelphia & Desulfurization, naphtha/reformer feed & 78000 \\
\hline Philadelphia energy solutions & Philadelphia & Desulfurization, naphtha/reformer feed & 78000 \\
\hline Philadelphia energy solutions & Philadelphia & Desulfurization, other distillate & 157000 \\
\hline Philadelphia energy solutions & Philadelphia & Desulfurization, other distillate & 157000 \\
\hline Philadelphia energy solutions & Philadelphia & Isomerization (isobutane) & 3800 \\
\hline Philadelphia energy solutions & Philadelphia & Isomerization (isobutane) & 3800 \\
\hline Philadelphia energy solutions & Philadelphia & Operating capacity & 335000 \\
\hline Philadelphia energy solutions & Philadelphia & Operating capacity & 350000 \\
\hline Philadelphia energy solutions & Philadelphia & Sulfur (short tons/day) & 76 \\
\hline Philadelphia energy solutions & Philadelphia & Sulfur (short tons/day) & 76 \\
\hline Philadelphia energy solutions & Philadelphia & Total oper cap (projected, next year) & 350000 \\
\hline Philadelphia energy solutions & Philadelphia & Total operable capacity & 335000 \\
\hline Philadelphia energy solutions & Philadelphia & Total operable capacity & 350000 \\
\hline Philadelphia energy solutions & Philadelphia & Vacuum distillation & 163200 \\
\hline Philadelphia energy solutions & Philadelphia & Vacuum distillation & 163200 \\
\hline Phillips 66 company & Linden & Alkylates & 18800 \\
\hline Phillips 66 company & Linden & Alkylates & 18800 \\
\hline Phillips 66 company & Linden & Cat cracking: fresh feed & 128000 \\
\hline Phillips 66 company & Linden & Cat cracking: fresh feed & 145000 \\
\hline Phillips 66 company & Linden & Cat cracking: fresh feed & 145000 \\
\hline Phillips 66 company & Linden & Cat reforming: low pressure & 32300 \\
\hline Phillips 66 company & Linden & Cat reforming: low pressure & 35900 \\
\hline Phillips 66 company & Linden & Cat reforming: low pressure & 35900 \\
\hline Phillips 66 company & Linden & Desulfurization, diesel fuel & 108000 \\
\hline Phillips 66 company & Linden & Desulfurization, diesel fuel & 108000 \\
\hline Phillips 66 company & Linden & Desulfurization, naphtha/reformer feed & 65500 \\
\hline Phillips 66 company & Linden & Desulfurization, naphtha/reformer feed & 65500 \\
\hline Phillips 66 company & Linden & Desulfurization, other distillate & 17500 \\
\hline Phillips 66 company & Linden & Desulfurization, other distillate & 17500 \\
\hline Phillips 66 company & Linden & Fuels solvent deasphalting & 22000 \\
\hline Phillips 66 company & Linden & Fuels solvent deasphalting & 22000 \\
\hline
\end{tabular}




\begin{tabular}{|c|c|c|c|}
\hline Phillips 66 company & Linden & Hydrogen (mmcfd) & 22 \\
\hline Phillips 66 company & Linden & Hydrogen (mmcfd) & 22 \\
\hline Phillips 66 company & Linden & Isomerization (isobutane) & 4000 \\
\hline Phillips 66 company & Linden & Isomerization (isobutane) & 4000 \\
\hline Phillips 66 company & Linden & Operating capacity & 258500 \\
\hline Phillips 66 company & Linden & Operating capacity & 272100 \\
\hline Phillips 66 company & Linden & Total oper cap (projected, next year) & 272100 \\
\hline Phillips 66 company & Linden & Total operable capacity & 258500 \\
\hline Phillips 66 company & Linden & Total operable capacity & 272100 \\
\hline Phillips 66 company & Linden & Vacuum distillation & 75000 \\
\hline Phillips 66 company & Linden & Vacuum distillation & 75000 \\
\hline United refining co & Warren & Alkylates & 4500 \\
\hline United refining co & Warren & Alkylates & 4500 \\
\hline United refining co & Warren & Asphalt \& road oil & 22000 \\
\hline United refining co & Warren & Asphalt \& road oil & 22000 \\
\hline United refining co & Warren & Cat cracking: fresh feed & 24000 \\
\hline United refining co & Warren & Cat cracking: fresh feed & 25000 \\
\hline United refining co & Warren & Cat cracking: fresh feed & 25000 \\
\hline United refining co & Warren & Cat cracking: recycled feed & 1000 \\
\hline United refining co & Warren & Cat cracking: recycled feed & 1000 \\
\hline United refining co & Warren & Cat reforming: high pressure & 13000 \\
\hline United refining co & Warren & Cat reforming: high pressure & 14000 \\
\hline United refining co & Warren & Cat reforming: high pressure & 14000 \\
\hline United refining co & Warren & Desulfurization, diesel fuel & 17000 \\
\hline United refining co & Warren & Desulfurization, diesel fuel & 17000 \\
\hline United refining co & Warren & Desulfurization, gasoline & 5000 \\
\hline United refining co & Warren & Desulfurization, gasoline & 5000 \\
\hline United refining co & Warren & Desulfurization, kerosene and jet & 5000 \\
\hline United refining co & Warren & Desulfurization, kerosene and jet & 5000 \\
\hline United refining co & Warren & Desulfurization, naphtha/reformer feed & 26000 \\
\hline United refining co & Warren & Desulfurization, naphtha/reformer feed & 26000 \\
\hline United refining co & Warren & Hydrogen (mmcfd) & 10 \\
\hline United refining co & Warren & Hydrogen (mmcfd) & 10 \\
\hline United refining co & Warren & Isomerization (isopentane/isohexane) & 8500 \\
\hline United refining co & Warren & Isomerization (isopentane/isohexane) & 8500 \\
\hline United refining co & Warren & Operating capacity & 65000 \\
\hline United refining co & Warren & Operating capacity & 70000 \\
\hline United refining co & Warren & Sulfur (short tons/day) & 67 \\
\hline United refining co & Warren & Sulfur (short tons/day) & 67 \\
\hline United refining co & Warren & Total oper cap (projected, next year) & 70000 \\
\hline United refining co & Warren & Total operable capacity & 65000 \\
\hline United refining co & Warren & Total operable capacity & 70000 \\
\hline United refining co & Warren & Vacuum distillation & 40000 \\
\hline
\end{tabular}




\section{References}

(1) Bruun, E. W.; Ambus, P.; Egsgaard, H.; Hauggaard-Nielsen, H., Effects of slow and fast pyrolysis biochar on soil C and N turnover dynamics. Soil Biol. Biochem. 2012, 46, 73-79, DOI: 10.1016/j.soilbio.2011.11.019.

(2) Ding, Y.; Liu, Y. G.; Liu, S. B.; Li, Z. W.; Tan, X. F.; Huang, X. X.; Zeng, G. M.; Zhou, L.; Zheng, B. H., Biochar to improve soil fertility. A review. Agron. Sustainable Dev. 2016, 36 (2), DOI: 10.1007/s13593-016-0372-z.

(3) Peters, J. F.; Iribarren, D.; Dufour, J., Biomass Pyrolysis for Biochar or Energy Applications? A Life Cycle Assessment. Environ. Sci. Technol. 2015, 49 (8), 5195-5202, DOI: 10.1021/es5060786.

(4) Ahrenfeldt, J.; Thomsen, T. P.; Henriksen, U.; Clausen, L. R., Biomass gasification cogeneration - A review of state of the art technology and near future perspectives. Appl. Therm. Eng. 2013, 50 (2), 1407-1417, DOI: 10.1016/j.applthermaleng.2011.12.040.

(5) Toor, S. S.; Rosendahl, L.; Rudolf, A., Hydrothermal liquefaction of biomass: A review of subcritical water technologies. Energy 2011, 36 (5), 2328-2342, DOI:

10.1016/j.energy.2011.03.013.

(6) Funke, A.; Ziegler, F., Hydrothermal carbonization of biomass: A summary and discussion of chemical mechanisms for process engineering. Biofuels, Bioprod. Biorefin. 2010, 4 (2), 160177, DOI: 10.1002/bbb.198.

(7) Posmanik, R.; Labatut, R. A.; Kim, A. H.; Usack, J. G.; Tester, J. W.; Angenent, L. T., Coupling hydrothermal liquefaction and anaerobic digestion for energy valorization from model biomass feedstocks. Bioresour. Technol. 2017, 233, 134-143, DOI:

10.1016/j.biortech.2017.02.095.

(8) Cavalaglio, G.; Coccia, V.; Cotana, F.; Gelosia, M.; Nicolini, A.; Petrozzi, A., Energy from poultry waste: An Aspen Plus-based approach to the thermo-chemical processes. Waste Manage. 2018, 73, 496-503, DOI: 10.1016/j.wasman.2017.05.037.

(9) Kantarli, I. C.; Stefanidis, S. D.; Kalogiannis, K. G.; Lappas, A. A., Utilisation of poultry industry wastes for liquid biofuel production via thermal and catalytic fast pyrolysis. Waste Manage. Res. 2019, 37 (2), 157-167, DOI: 10.1177/0734242x18799870.

(10) Agblevor, F. A.; Beis, S.; Kim, S. S.; Tarrant, R.; Mante, N. O., Biocrude oils from the fast pyrolysis of poultry litter and hardwood. Waste Manage. 2010, 30 (2), 298-307, DOI:

10.1016/j.wasman.2009.09.042.

(11) Sharpley, A.; Slaton, N.; Tabler, T.; VanDevender, K.; Daniels, M.; Jones, F.; Daniel, T. Nutrient analysis of poultry litter; University of Arkansas System: 2009.

(12) Bridgwater, A. V., Review of fast pyrolysis of biomass and product upgrading. Biomass Bioenergy 2012, 38, 68-94, DOI: 10.1016/j.biombioe.2011.01.048.

(13) Jones, S. B.; Zhu, Y.; Anderson, D. B.; Hallen, R. T.; Elliott, D. C.; Schmidt, A. J.; Albrecht, K. O.; Hart, T. R.; Butcher, M. G.; Drennan, C.; Snowden-Swan, L. J.; Davis, R.; Kinchin, C. Process Design and Economics for the Conversion of Algal Biomass to Hydrocarbons: Whole Algae Hydrothermal Liquefaction and Upgrading; PNNL-23227; Pacific Northwest National Lab. (PNNL): Richland, WA, 2014.

(14) Tews, I. J.; Zhu, Y.; Drennan, C.; Elliott, D. C.; Snowden-Swan, L. J.; Onarheim, K.; Solantausta, Y.; Beckman, D. Biomass Direct Liquefaction Options. TechnoEconomic and Life Cycle Assessment; PNNL-23579; Pacific Northwest National Lab. (PNNL): Richland, WA, 2014. 
(15) Swanson, R. M.; Platon, A.; Satrio, J. A.; Brown, R. C.; Hsu, D. D. Techno-Economic Analysis of Biofuels Production Based on Gasification; NREL/TP-6A20-46587; National Renewable Energy Lab. (NREL): Golden, CO, 2010.

(16) Baniasadi, M.; Tugnoli, A.; Conti, R.; Torri, C.; Fabbri, D.; Cozzani, V., Waste to energy valorization of poultry litter by slow pyrolysis. Renewable Energy 2016, 90, 458-468, DOI: 10.1016/j.renene.2016.01.018.

(17) Di Blasi, C., Modeling chemical and physical processes of wood and biomass pyrolysis. Prog. Energy Combust. Sci. 2008, 34 (1), 47-90, DOI: 10.1016/j.pecs.2006.12.001.

(18) Al Arni, S., Comparison of slow and fast pyrolysis for converting biomass into fuel. Renewable Energy 2018, 124, 197-201, DOI: 10.1016/j.renene.2017.04.060.

(19) Brown, T. R.; Wright, M. M.; Brown, R. C., Estimating profitability of two biochar production scenarios: slow pyrolysis vs fast pyrolysis. Biofuels, Bioprod. Biorefin. 2011, 5 (1), 54-68, DOI: 10.1002/bbb.254.

(20) Wright, M. M.; Satrio, J. A.; Brown, R. C.; Daugaard, D. E.; Hsu, D. D. Techno-Economic Analysis of Biomass Fast Pyrolysis to Transportation Fuels; NREL/TP-6A20-46586; National Renewable Energy Lab. (NREL): Golden, CO, 2010.

(21) Kim, S. S.; Agblevor, F. A.; Lim, J., Fast pyrolysis of chicken litter and turkey litter in a fluidized bed reactor. J. Ind. Eng. Chem. 2009, 15 (2), 247-252, DOI:

10.1016/j.jiec.2008.10.004.

(22) Kim, S. S.; Agblevor, F. A., Pyrolysis characteristics and kinetics of chicken litter. Waste Manage. 2007, 27 (1), 135-140, DOI: 10.1016/j.wasman.2006.01.012.

(23) Hussein, M. S.; Burra, K. G.; Amano, R. S.; Gupta, A. K., Temperature and gasifying media effects on chicken manure pyrolysis and gasification. Fuel 2017, 202, 36-45, DOI: 10.1016/j.fuel.2017.04.017.

(24) Hussein, M. S.; Burra, K. G.; Amano, R. S.; Gupta, A. K., Effect of oxygen addition in steam gasification of chicken manure. Fuel 2017, 189, 428-435, DOI:

10.1016/j.fuel.2016.11.005.

(25) Devi, L.; Ptasinski, K. J.; Janssen, F., A review of the primary measures for tar elimination in biomass gasification processes. Biomass Bioenergy 2003, 24 (2), 125-140, DOI:

10.1016/s0961-9534(02)00102-2.

(26) Font-Palma, C., Characterisation, kinetics and modelling of gasification of poultry manure and litter: An overview. Energy Convers. Manage. 2012, 53 (1), 92-98, DOI:

10.1016/j.enconman.2011.08.017.

(27) Horvat, A.; Pandey, D. S.; Kwapinska, M.; Mello, B. B.; Gomez-Barea, A.; Fryda, L. E.;

Rabou, L.; Kwapinski, W.; Leahy, J. J., Tar yield and composition from poultry litter gasification in a fluidised bed reactor: effects of equivalence ratio, temperature and limestone addition. RSC Adv. 2019, 9 (23), 13283-13296, DOI: 10.1039/c9ra02548k.

(28) Jones, S. B.; Valkenburt, C.; Walton, C. W.; Elliott, D. C.; Holladay, J. E.; Stevens, D. J.; Kinchin, C.; Czernik, S. Production of Gasoline and Diesel from Biomass via Fast Pyrolysis, Hydrotreating and Hydrocracking: A Design Case; PNNL-18284 Rev. 1; Pacific Northwest National Lab. (PNNL), Richland, WA (United States): 2009.

(29) Baliban, R. C.; Elia, J. A.; Floudas, C. A.; Xiao, X.; Zhang, Z. J.; Li, J.; Cao, H. B.; Ma, J.; Qiao, Y.; Hu, X. T., Thermochemical Conversion of Duckweed Biomass to Gasoline, Diesel, and Jet Fuel: Process Synthesis and Global Optimization. Ind. Eng. Chem. Res. 2013, 52 (33), 11436-11450, DOI: 10.1021/ie3034703. 
(30) Aspen Plus. Getting Started Modeling Processes with Solids; Aspen Technology, Inc.: Cambridge, MA, 2013.

(31) Peters, J. F.; Banks, S. W.; Bridgwater, A. V.; Dufour, J., A kinetic reaction model for biomass pyrolysis processes in Aspen Plus. Appl. Energy 2017, 188, 595-603, DOI:

10.1016/j.apenergy.2016.12.030.

(32) U.S. States: State Profiles and Energy Estimates - U.S. Energy Information Administration (EIA). https://www.eia.gov/state/maps.php (accessed 2019-08).

(33) Bridgwater, A. V., The production of biofuels and renewable chemicals by fast pyrolysis of biomass. Int. J. Global Energy Issues 2007, 27 (2), 160-203.

(34) Freeman, C. J.; Jones, S. B.; Padmaperuma, A. B.; Santosa, D. M.; Valkenburg, C.; Shinn, J. Initial Assessment of U.S. Refineries for Purposes of Potential Bio-Based Oil Insertions; PNNL-22432; Pacific Northwest National Lab. (PNNL), Richland, WA, United States: 2013. (35) Zhang, Q.; Gong, J.; Skwarczek, M.; Yue, D. J.; You, F. Q., Sustainable Process Design and Synthesis of Hydrocarbon Biorefinery through Fast Pyrolysis and Hydroprocessing. AIChE J. 2014, 60 (3), 980-994, DOI: 10.1002/aic.14344.

(36) Aden, A.; Ruth, M.; Ibsen, K.; Jechura, J.; Neeves, K.; Sheehan, J.; Wallace, R. Lignocellulosic biomass to ethanol process design and economics utilizing co-current dilute acid prehydrolysis and enzymatic hydrolysis for corn stover; NREL report NREL/TP-510-32438: 2002.

(37) Phillips, S.; Aden, A.; Jechura, J.; Dayton, D. Thermochemical ethanol via indirect gasification and mixed alcohol synthesis of lignocellulosic biomass.; National Renewable Energy Laboratory: Golden CO, 2007.

(38) Roberts, K. G.; Gloy, B. A.; Joseph, S.; Scott, N. R.; Lehmann, J., Life Cycle Assessment of Biochar Systems: Estimating the Energetic, Economic, and Climate Change Potential. Environ. Sci. Technol. 2010, 44 (2), 827-833, DOI: 10.1021/es902266r.

(39) Aspen Capital Cost Estimator - User's Guide (V8.0); Aspen Technology, Inc.: Burlington, MA, 2012.

(40) Monthly Average Retail Price of Electricity - Industrial - New York State Energy Research and Development Authority (NYSERDA). https://www.nyserda.ny.gov/Researchers-andPolicymakers/Energy-Prices/Electricity/Monthly-Avg-Electricity-Industrial (accessed 2020-01). (41) Monthly Average Price of Natural Gas - Industrial - New York State Energy Research and Development Authority (NYSERDA). https://www.nyserda.ny.gov/Researchers-andPolicymakers/Energy-Prices/Natural-Gas/Monthly-Average-Price-of-Natural-Gas-Industrial (accessed 2020-01).

(42) Weekly On-Highway Diesel Prices - New York State Energy Research and Development Authority (NYSERDA). https://www.nyserda.ny.gov/Researchers-and-Policymakers/EnergyPrices/On-Highway-Diesel/Weekly-Diesel-Prices (accessed 2019-08).

(43) Weekly Average Motor Gasoline Prices - New York State Energy Research and Development Authority (NYSERDA). https://www.nyserda.ny.gov/Researchers-andPolicymakers/Energy-Prices/Motor-Gasoline/Weekly-Average-Motor-Gasoline-Prices (accessed 2019-08).

(44) New York State Energy Profile; U.S. Energy Information Administration (EIA).

(45) Huang, Y.; Anderson, M.; McIlveen-Wright, D.; Lyons, G. A.; McRoberts, W. C.; Wang, Y. D.; Roskilly, A. P.; Hewitt, N. J., Biochar and renewable energy generation from poultry litter waste: A technical and economic analysis based on computational simulations. Appl. Energy 2015, 160, 656-663, DOI: 10.1016/j.apenergy.2015.01.029. 
(46) Myhre, G.; Shindell, D.; Bréon, F.-M.; Collins, W.; Fuglestvedt, J.; Huang, J.; Koch, D.; Lamarque, J.-F.; Lee, D.; Mendoza, B.; Nakajima, T.; Robock, A.; Stephens, G.; Takemura, T.; Zhang, H. Anthropogenic and Natural Radiative Forcing; IPCC: New York, NY, USA, 2013.

(47) USDA Natural Resources Conservation Service: Animal Feeding Operations.

https://www.nrcs.usda.gov/wps/portal/nrcs/main/national/plantsanimals/livestock/afo/ (accessed 2020-01). 